
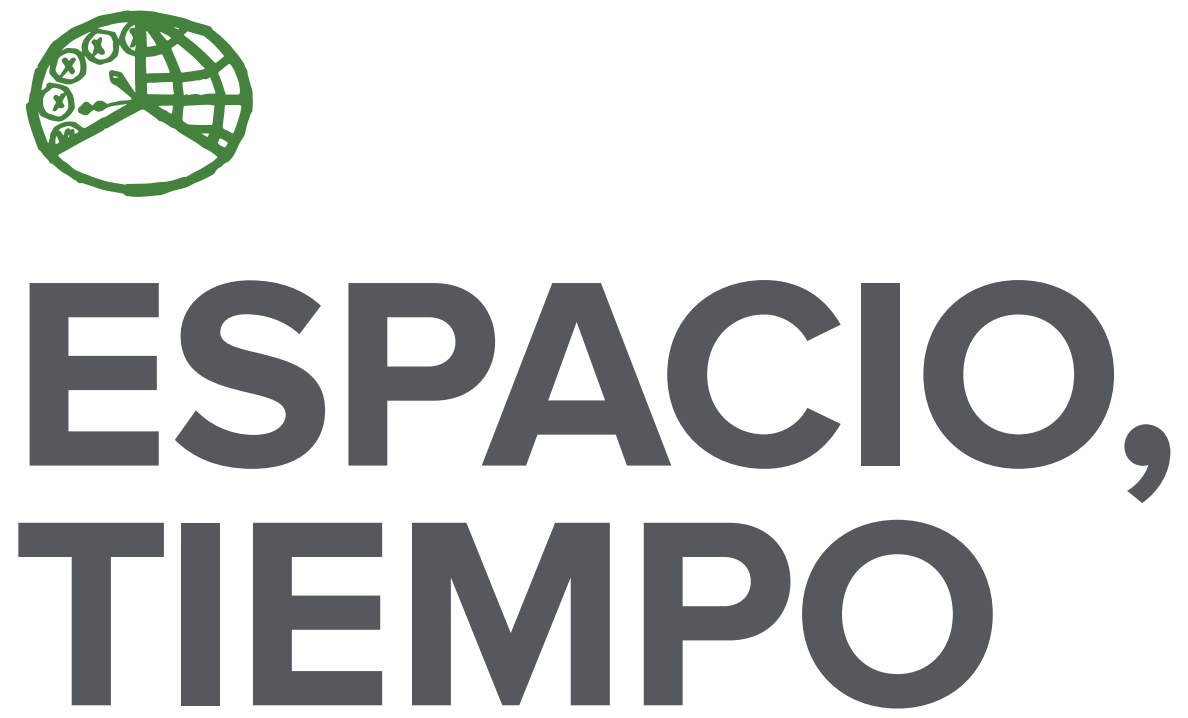

AÑO 2021

ISSN 1130-2968

E-ISSN 2340-146X
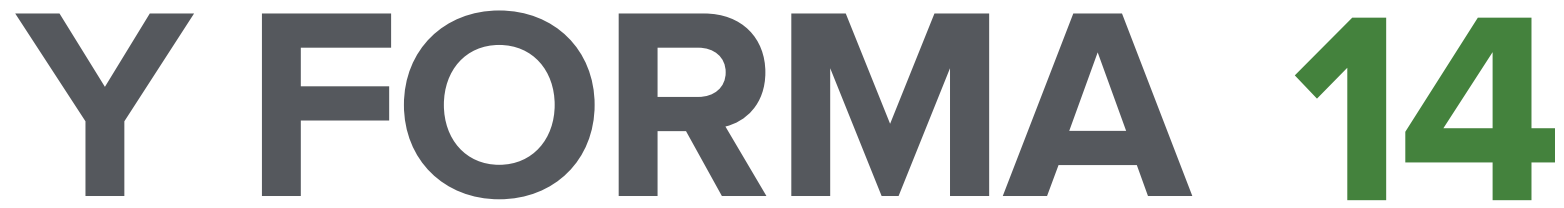

SERIE VI GEOGRAFİA

REVISTA DE LA FACULTAD DE GEOGRAFÍA E HISTORIA 


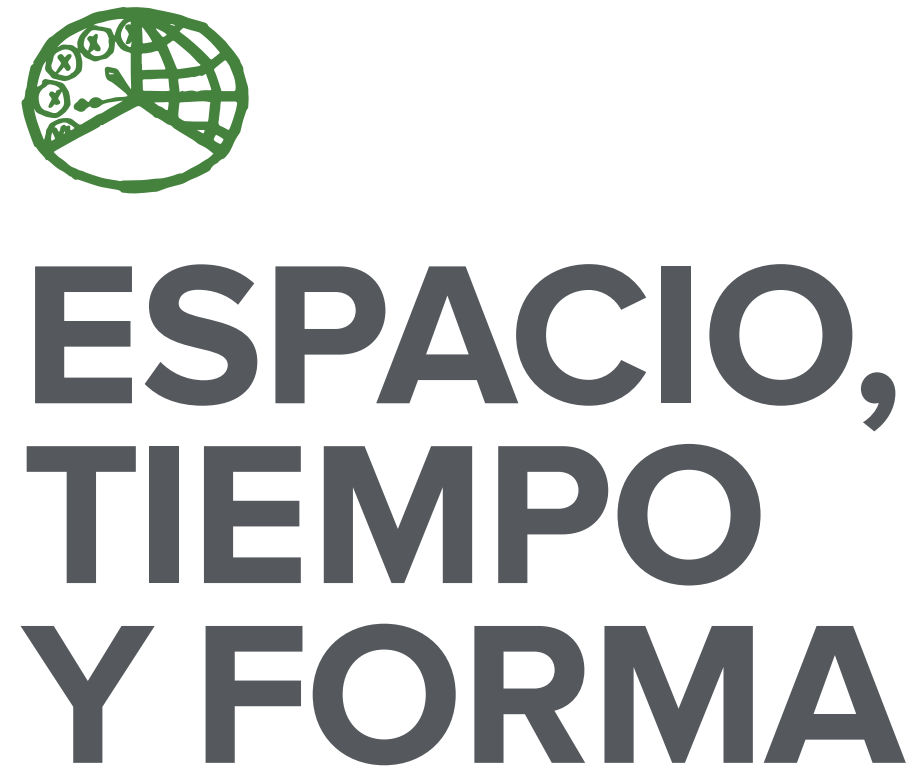

AÑO 2021

ISSN 1130-2968

E-ISSN 2340-146X

SERIE VI GEOGRAFÍA

REVISTA DE LA FACULTAD DE GEOGRAFİA E HISTORIA

DOI: https://doi.org/10.5944/etfvi.14.2021

\section{UחED}

UNIVERSIDAD NACIONAL DE EDUCACIÓN A DISTANCIA 
La revista Espacio, Tiempo y Forma (siglas recomendadas: ETF), de la Facultad de Geografía e Historia de la UNED, que inició su publicación el año 1988, está organizada de la siguiente forma:

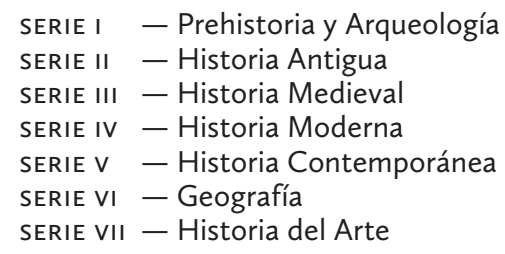

Excepcionalmente, algunos volúmenes del año 1988 atienden a la siguiente numeración:

$$
\begin{aligned}
& \mathrm{N}^{\circ} 1 \text { - Historia Contemporánea } \\
& \mathrm{N}^{\circ} 2 \text { - Historia del Arte } \\
& \mathrm{N}^{\circ} 3 \text { - Geografía } \\
& \mathrm{N} .^{\circ} 4 \text { - Historia Moderna }
\end{aligned}
$$

ETF no se solidariza necesariamente con las opiniones expresadas por los autores.

\author{
UNIVERSIDAD NACIONAL DE EDUCACIÓN A DISTANCIA \\ Madrid, 2020 \\ SERIE VI - GEOGRAFÍA N. ${ }^{\circ} 14,2021$ \\ ISSN 1130-2968 - E-ISSN 2340-146x \\ DEPÓSITO LEGAL \\ $M-21.037-1988$ \\ URL \\ ETF VI · GEOGRAFÍA · http://revistas.uned.es/index.php/ETFVI \\ DISEÑO Y COMPOSICIÓN \\ Carmen Chincoa Gallardo · http://www.laurisilva.net/cch \\ Impreso en España · Printed in Spain
}




\section{ARTÍCULOS · ARTICLES}





\title{
INVESTIGACIÓN Y ENSEÑANZA EN DIDÁCTICA DE LA CLIMATOLOGÍA. ESTADO DE LA CUESTIÓN (1980-2020) (ESPAÑA)
}

\author{
RESEARCH AND TEACHING IN \\ DIDACTIC OF CLIMATOLOGY. STATE \\ OF THE ART (1980-2020) (SPAIN)
}

\author{
Álvaro-Francisco Morote Seguido ${ }^{1}$ \\ Recibido: 10/08/2021 - Aceptado: 28/09/2021 \\ DOI: http://doi.org/10.5944/etfvi.14.2021.31332
}

\section{Resumen ${ }^{2}$}

El objetivo de este trabajo es realizar un estado de la cuestión de las contribuciones realizadas en España sobre la investigación y enseñanza en Didáctica de la Climatología (1980-2020). Este trabajo resulta de notable interés para comprobar la evolución y temáticas de estas publicaciones tras el trascurso de cuatro décadas desde la formación del grupo de trabajo de Didáctica de la Geografía (I986). A partir de la consulta de diferentes bases de datos (8.303 publicaciones identificadas) y tras su posterior depuración, la cifra de trabajos ascendió a 89. Las contribuciones se distribuyen en dos ejes principales: trabajos sobre el clima y tiempo atmosférico $(53,9 \%)$ y sobre el cambio climático $(46,1 \%)$, siendo este último eje el que mayor atención ha tenido en la última década. A su vez, se han identificado 3 temáticas: trabajos sobre la enseñanza; análisis de las representaciones sociales; y libros de texto. Como conclusión, cabe indicar que la investigación y enseñanza en Didáctica de la Climatología en España es un tema del s. XXI y, a esperas de conocer a corto plazo la tendencia de estas investigaciones y temáticas teniendo en cuenta la influencia de los Objetivos de Desarrollo Sostenible (Agenda 2030) y el cambio climático.

\section{Palabras clave}

Climatología; Didáctica; Geografía; España.

\section{Abstract}

The aim of this research is to carry out a state of the art of the contributions made in Spain on research and teaching in Didactic of Climatology (1980-2020). This

1. Departamento de Didáctica de las Ciencias Experimentales y Sociales (Universidad de Valencia); alvaro.morote@uv.es; https://orcid.org/oooo-0003-2438-4961

2. Esta investigación se inserta en el proyecto «Las representaciones sociales de los contenidos escolares en el desarrollo de las competencias docentes» (PGC2018-094491-B-C32) financiado por el Ministerio de Ciencia, Innovación y Universidades y cofinanciado con fondos FEDER de la UE. 
work is interesting in order to check the evolution and themes of these publications after four decades have elapsed since the formation of the Geography Didactic group (I986). From the consultation of different databases $(8,303$ publications identified) and after their subsequent filtering, the number of works rose to 89 . The contributions are distributed in two main groups: works on climate and atmospheric weather (53.9\%); and on climate change (46.I\%). This last group being the one that has received the most attention in the last decade. In turn, 3 themes have been identified: works on teaching; analysis of social representations; and school textbooks. In conclusion, it should be noted that research and teaching in Didactic of Climatology in Spain is a subject of the s. XXI and, waiting to know in the short term the trend of these investigations and topics, taking into account the influence of the Sustainable Development Goals (Agenda 2030) and climate change.

\section{Keywords}

Climatology; Didactics; Geography; Spain. 


\section{INTRODUCCIÓN}

En la actualidad, la Climatología ha cobrado un interés notable en el ámbito educativo por sus implicaciones en la sociedad (Cruz, 20ıо; Da Silva \& Cardoso, 20I9; Martínez-Medina \& López-Fernández, 2016; Olcina, 20I7). Respecto a la definición de Climatología, Gil \& Olcina (20I7) explican que Climatología, clima y tiempo atmosférico son conceptos muy afines, pero, asimismo, distintos y diversos. Para el caso del primero no hay una definición unánimemente compartida, siendo la más sencilla y menos comprometida la de ciencia de los climas. Asimismo, Gil \& Olcina (20I7) indican que entre Climatología y Meteorología existen rasgos diferenciadores: los que se dedican a la Meteorología han sido y son físicos (meteorólogos); los de la Climatología, geógrafos (climatólogos). El objeto, por tanto, de los segundos es analizar la sucesión habitual o ritmo de esos estados de la atmósfera. En cuanto al clima, Martínez-Fernández \& Olcina (2019, p. I25) explican que «el clima es el conjunto de mecanismos y procesos atmosféricos percibidos y vividos por el ser humano (el ambiente permanente que se da en un lugar concreto) más la explicación de sus causas (la sucesión de tipos de tiempo)».

Respecto al enfoque didáctico tradicional, Sánchez-Ogallar (200o, p. 484) argumenta que este «viene siendo la consideración del clima como conjunto de condiciones de la atmósfera que caracterizan a un lugar determinado a partir del balance medio obtenido de la sucesión de una serie de estados del tiempo». En el ámbito educativo, Olcina (2017) ha puesto de manifiesto la complejidad de la explicación de los contenidos sobre el clima y especialmente, los vinculados con el cambio climático debido a la multitud de variables que interaccionan. Incluso en la actualidad a los científicos no les resulta fácil llevar a cabo una correcta explicación (Martínez-Fernández \& Olcina, 2019). Por su parte, Sánchez-Ogallar (2000) explica que «la comprensión por el alumnado de este megaconcepto de la Geografía resulta problemático, pues requiere engarzar una serie de conceptos de menor jerarquía, complejos en sí mismos y enlazados por relaciones de causa-efecto» (p. 484).

En España, la enseñanza tiempo atmosférico y el clima constituye un contenido que se debe tratar prácticamente en todas las etapas escolares (Martínez-Fernández \& Olcina, 20I9; Morote \& Olcina, 202Ia; Sebastiá \& Tonda, 2018; Valbuena \& Valverde, 2006). Martínez-Fernández \& Olcina (2019) indican que en la educación obligatoria (Educación Primaria y Secundaria), la enseñanza de la Climatología adquiere:

«un aceptable (aunque siempre mejorable) tratamiento como contenidos curriculares y, por ende, en el desarrollo y estructuración de las programaciones, constituyendo un saber muy útil y práctico con enorme importancia, además, en muchos aspectos de la vida cotidiana de los alumnos» (p. 146).

De ahí, como indican estos autores, la importancia dedicada al logro de un aprendizaje significativo, mientras que Tonda \& Sebastiá (2003) destacan el saber útil y necesario del conocimiento y comprensión del tiempo atmosférico. Autores como Soñora, Rodríguez \& Troitiño (2009) argumentan el interés por la educación ambiental, ya que todos los profesionales de la enseñanza tienen muy presente que 
esta temática es una parte esencial de la formación holística de la ciudadanía del s. XXI (Souto, 2018).

Otros aspectos que denotan un mayor interés a esta temática es el cambio climático (Carman et al., 2020; Namdar, 2018) debido, especialmente, a los riesgos atmosféricos asociados que están provocando cada vez más, víctimas humanas y perdidas económicas (Pérez-Morales, Gil \& Quesada, 202I). Por ejemplo, fenómenos como los episodios de precipitaciones intensas y las sequías, según indican los informes de cambio climático, serán más intensos y frecuentes (Centro de Estudios y Experimentación de Obras Públicas [CEDEX], 20I7; Intergovernmental Panel on Climate Change [IPCC], 202I). Al respecto, cabría hacer mención a las graves y recientes inundaciones sufridas en julio de 202I en Centro Europa y Asia con centenares de fallecidos. Por tanto, la enseñanza del clima y el cambio climático es un desafío en el ámbito educativo ya que resulta necesario formar a la sociedad y a la población más joven en la adaptación y comprensión sobre este problema socioambiental (Morote \& Olcina, 202rb).

Caride \& Meira (2019) explican que:

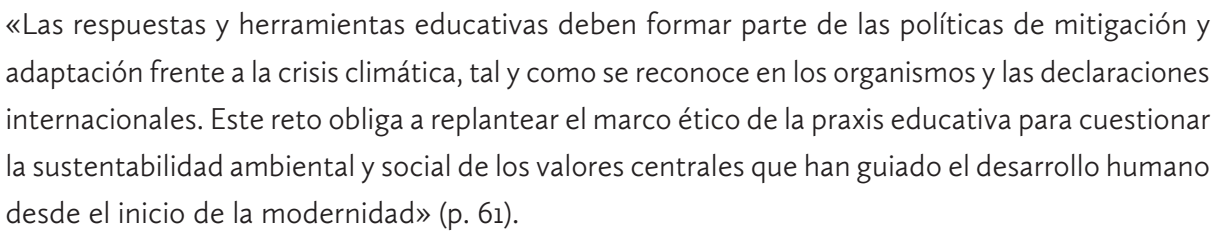

Al respecto, con la celebración de la Conferencia de las Naciones Unidas sobre el cambio climático (COP25 Madrid 2019), la Ministra de Educación, Cultura y Deporte planteó la posibilidad de crear una asignatura para tratar este fenómeno en la etapa escolar. No obstante, y como manifestaron en una declaración conjunta el Colegio de Geógrafos de España y la Asociación Española de Geografía (20I9), las cuestiones sobre el cambio climático ya se enseñan en la etapa escolar. Morote \& Olcina (2020) explican que otra cosa es que la formación que se está llevando a cabo sobre esta temática sea escasa e inadecuada, siendo necesario repensar el currículum actual de Geografía y Ciencias Sociales. Asimismo, estos autores apuestan por llevar una mejor y mayor formación de los/as docentes. También cabe destacar que en España, por vez primera, se ha aprobado una legislación nacional en materia de cambio climático (Ley de Cambio Climático; mayo de 202I) en la que se dedica una sección a la enseñanza de este fenómeno (Título VIII «Educación, Investigación e Innovación en la lucha contra el cambio climático y la transición energética»).

Este marco normativo ha sido respaldado por diferentes organismos internacionales. Por ejemplo, el Quinto Informe de Cambio Climático del IPCC (IPCC, 20I4) ya dejó constancia de la importancia de la enseñanza del cambio climático. Asimismo, cabe destacar el interés mostrado por las Naciones Unidas por una enseñanza rigurosa sobre los riesgos derivados de este fenómeno en el ámbito educativo, como eje de acción para lograr los Objetivos de Desarrollo Sostenible (ODS) (Agenda 2030) y más específicamente con el objetivo $\mathrm{n}^{\circ} \mathrm{I} 3$ «Acción por el clima» (United Nations, 2015). También, este respaldo ha sido manifestado por 
diferentes autores en el ámbito español (Morote \& Olcina, 2020; 202Ia) dando importancia a la enseñanza como una de las acciones más necesarias para adaptarse a este fenómeno y sus efectos asociados.

El interés que motiva la realización de este trabajo se debe también al reducido número de investigaciones que se han realizado tanto en el contexto español como internacional sobre el estado de la cuestión de publicaciones sobre Didáctica de la Climatología. En España, cabría citar como investigación previa el trabajo de Tonda \& Sebastiá (2003) en el que se realizó una aproximación de las contribuciones realizadas hasta ese momento (comienzos del s. XXI). Estos autores analizaron como hasta el 2003 la cifra de publicaciones no llegaba a la decena. Existen, también, trabajos recientes en los que se referencian diferentes publicaciones para completar el marco teórico (ver Morote, 2020a). Por ejemplo, estas contribuciones desde la Didáctica de la Geografía se relacionan con la dificultad de enseñar Climatología (Tonda \& Sebastiá, 2003), metodologías para enseñar la Climatología local (Valbuena \& Valverde, 2006), el uso del climograma (Martínez-Romera, 2013), refranes (MartínezIbarra, Arias \& Gómez, 20I6), análisis curricular y textos literarios (García de la Vega, 20I6), propuestas de centros de interpretación (Morote \& Moltó, 20I7), la enseñanza y análisis de libros texto (Educación Primaria) (Martínez-Medina \& López-Fernández, 2016; Morote \& Olcina, 2020), la enseñanza en la Educación Primaria (MartínezFernández \& Olcina, 20I9), o la Educación Secundaria y Bachillerato (Olcina, 20I7).

En cuanto al ámbito internacional, las referencias en los últimos años ponen de manifiesto también el interés de la temática, especialmente aquellas que tienen que ver con el cambio climático, por ejemplo: en Centro América y Norteamérica (McWhirter \& Shealy, 2018; Li et al., 202I; Sezen-Barrie \& Marbach-Ad, 202I); Sudamérica (Da Rocha et al., 2020); Europa (Jeong et al., 202I; Kurup et al., 202I); África (Anyanwu \& Grange, 2017); y Asia y Oceanía (Ahmad \& Numan, 2015; Li \& Liu, 202I).

El objetivo de este trabajo es llevar a cabo para el caso español, un estado de la cuestión de las publicaciones sobre la investigación y enseñanza de la Didáctica de la Climatología (1980-2020). Tras cuatro décadas desde la formación del grupo de Didáctica de la Geografía (I986) de la Asociación Española de Geografía y otros hitos que se comentarán en el siguiente apartado, resulta necesario hacer un balance de la tendencia y estado actual de las publicaciones vinculadas con la Didáctica de la Climatología. Como hipótesis de partida se establece que la tendencia de las investigaciones habría aumentado en la última década por el interés del tema, especialmente por las contribuciones que tienen que ver con el cambio climático y sus efectos asociados. También, diferentes hitos como la realización de números especiales o dedicados a esta temática en revistas científicas, así como ejes temáticos en congresos sobre didáctica habría repercutido en el incremento de estas publicaciones.

\section{METODOLOGÍA}

Metodológicamente se ha seguido el procedimiento de trabajos previos vinculados con búsquedas bibliográficas en Didáctica de la Geografía (ver Morote, 202ob). Para ello, el primer paso ha sido la definición de diferentes descriptores (and/or), tanto en 
castellano e inglés: didáctica; educación; enseñanza; Climatología; clima; tiempo atmosférico; España; instruction; didactic; education; teaching; Climatologlogy; climate; atmospheric weather, Spain. En el proceso de búsqueda, estos descriptores debían reflejarse en el título, palabras clave y/o en el resumen de las publicaciones. En relación con los trabajos se ha tenido en cuenta los artículos de investigación, capítulos de libro, libros y actas de congreso. En cuanto al periodo de estudio se ha seleccionado el intervalo 1980-2020, debido al interés de analizar la evolución de las publicaciones objeto de estudio tras cuatro décadas por: I) la creación del Grupo de Didáctica de la Geografía de la AGE (I986); 2) la adscripción de la Didáctica de la Geografía al área de conocimiento de Didáctica de las Ciencias Sociales (Real Decreto de I984, en desarrollo de la Ley de reforma universitaria) (De Miguel, 2013); y 3) la incidencia de la creación de la revista Didáctica Geográfica (I977) (revista nacional referente de Didáctica de la Geografía). También, hasta esa fecha, como indican Sebastiá \& Tonda (2012), en los congresos de Geografía, todavía no se había prestado un interés especial a la enseñanza de la Geografía.

Tras definir las palabras clave y el marco temporal, se procedió en esta primera fase de búsqueda a la consulta de diferentes bases de datos: Dialnet, Scielo, Scopus y Web of Science. En estas se han tenido en cuenta los trabajos adscritos a las Ciencias Sociales. Algunas de las limitaciones de esta fase se han encontrado, por ejemplo, con la base de datos Dialnet ya que contemplan las actas de congresos como «artículos de libro» (capítulos de libro). En cuanto al descriptor «clima» cabe mencionar que el número de publicaciones resultante ha sido superior al resto ya que la mayoría de los trabajos se vinculaban con el «clima del aula» (publicaciones desde el ámbito educativo y pedagógico). En esta primera fase la cifra total de resultados obtenidos fue de 8.28I (ver Figura I).

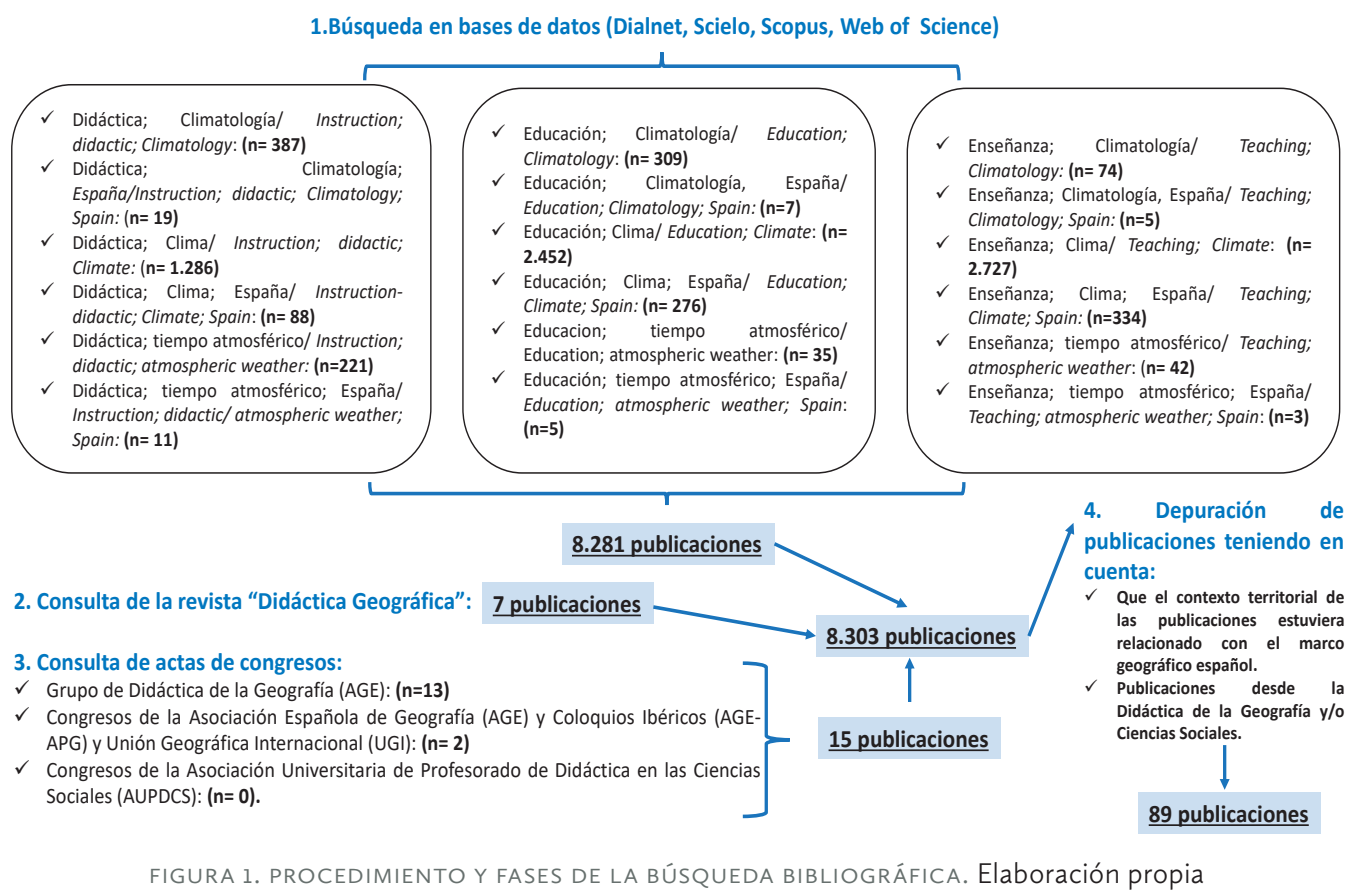


En segundo lugar, para completar los resultados obtenidos de la primera fase, se consultaron las publicaciones en la revista Didáctica Geográfica (los trabajos publicados desde 1977 están accesibles en la web). El número total de artículos relacionados con el tema objeto de estudio ha ascendido a 7. Como limitación de trabajo, cabe indicar que algunas publicaciones pueden no aparecer en las bases de datos consultadas, concretamente aquellas relacionadas con actas de congresos. Por este motivo, una tercera fase de búsqueda ha sido la revisión de estas actas en los congresos de: I) Grupo de Didáctica de la Geografía (AGE) (actas accesibles en la web desde I988); 2) Asociación Española de Geografía (AGE) (actas disponibles online desde 2013); 3) congresos Ibéricos de Geografía (AGE) y Asociación Portuguesa de Geografía (APG) (disponibles en la web desde 2002); 4) Unión Geográfica Internacional (UGI) (actas accesibles desde I998); y 5) Asociación Universitaria de Profesorado de Didáctica en las Ciencias Sociales (AUPDCS) (actas disponibles desde 1997). El trabajo de digitalización de estos trabajos por estas asociaciones en sus respectivas páginas webs (ver Anexo II) ha facilitado enormemente el rastreo de publicaciones que no se incluían en las bases de datos, siendo i5 la cifra total de investigaciones registradas.

Como resultado de estas tres fases, la cifra total de publicaciones ascendió a 8.303. A continuación, se procedió a revisar todas estas contribuciones y desechando aquellas que se repetían en las bases de datos. Para el proceso de depuración se han tenido en cuenta 2 criterios: I) que el contexto territorial de estos trabajos estuviera relacionado con el marco geográfico español; y 2) que las publicaciones se hayan realizado desde la Didáctica de la Geografía y/o Ciencias Sociales. No obstante, cabe advertir que también se han recopilado algunos trabajos realizados desde las Ciencias y desde las Ciencias de la Educación ya que, tras su consulta, se ha podido comprobar que tratan el tema objeto de estudio con cierta perspectiva geográfica y territorial, por ejemplo, aquellas publicaciones vinculadas con la enseñanza del cambio climático. Asimismo, a la hora de analizar las temáticas cabe destacar que algún trabajo se puede adscribir a varias (por ejemplo, investigaciones que hayan tratado tanto el clima como el cambio climático). Con este último paso de depuración, la cifra resultante de publicaciones ascendió a 89 . Se trata de una cifra similar que ha sido obtenida en otras investigaciones sobre Didáctica de la Geografía. Por ejemplo, Morote (2020b) en su trabajo sobre libros de texto identificó 80 trabajos en el periodo I980-20I9. Finalmente, cabe indicar que: I) en la Bibliografía sólo se han recogido los trabajos citados en el texto; y 2) todos los trabajos identificados se recogen en las tablas y en el Anexo $\mathrm{I}$.

\section{RESULTADOS}

En el periodo objeto de estudio (1980-2020) se han identificado un total de 89 publicaciones, siendo la tendencia de los trabajos ascendente, especialmente en la última década (20II-2020) (ver Figura 2). No es hasta principios del s. XXI cuando realmente esta línea de trabajo cobra interés entre los investigadores en el contexto español. Además, cabe advertir que se han diferenciado dos grandes ejes: I) trabajos 
vinculados con la Climatología en general (53,9\%; ( $\mathrm{n}=48)$; y 2) trabajos relacionados con el cambio climático (46,I\%; $\mathrm{n}=4 \mathrm{I})$. Pero de estos últimos cabe destacar que el $85,4 \%(n=35)$ han sido publicados en la última década (ver Figura 3).

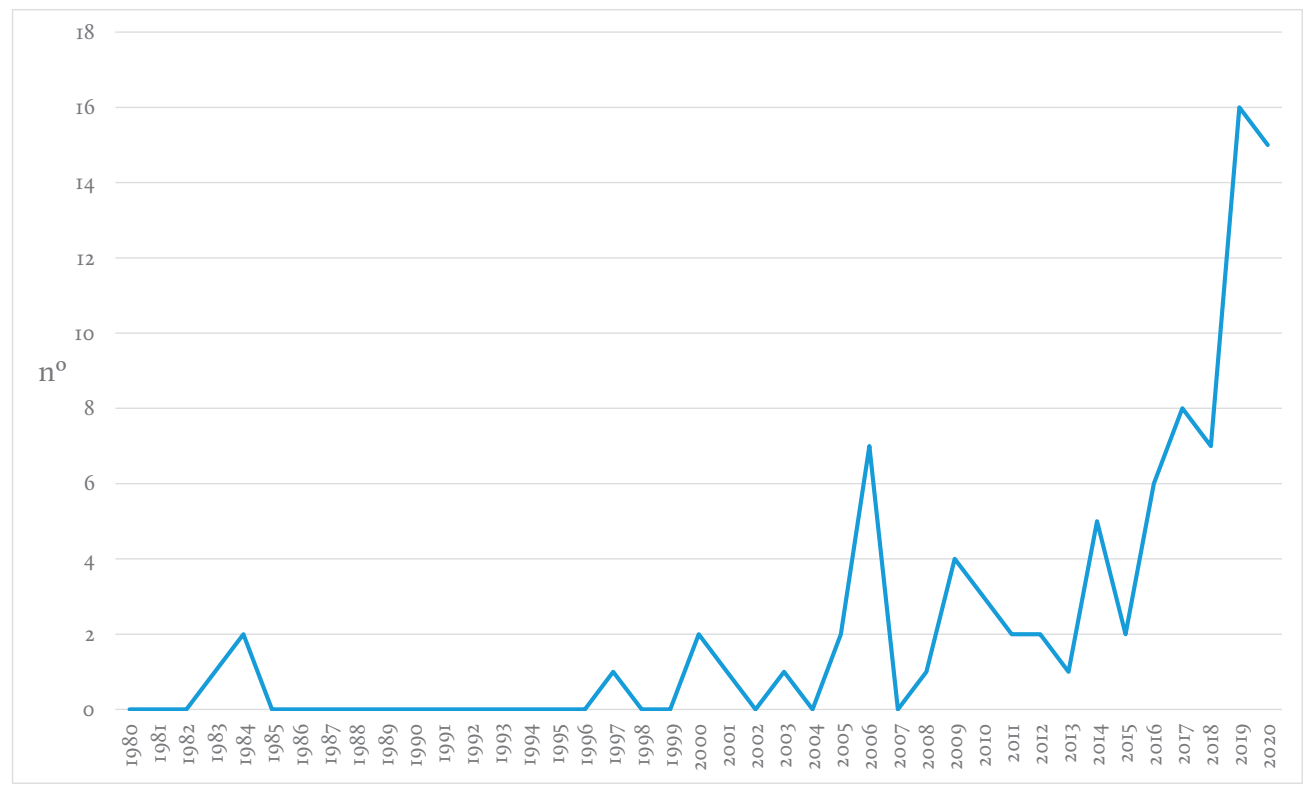

FIGURA 2. EVOLUCIÓN DE LAS PUBLICACIONES SOBRE DIDÁCTICA DE LA CLIMATOLOGÍA EN ESPAÑA (19802020). Elaboración propia

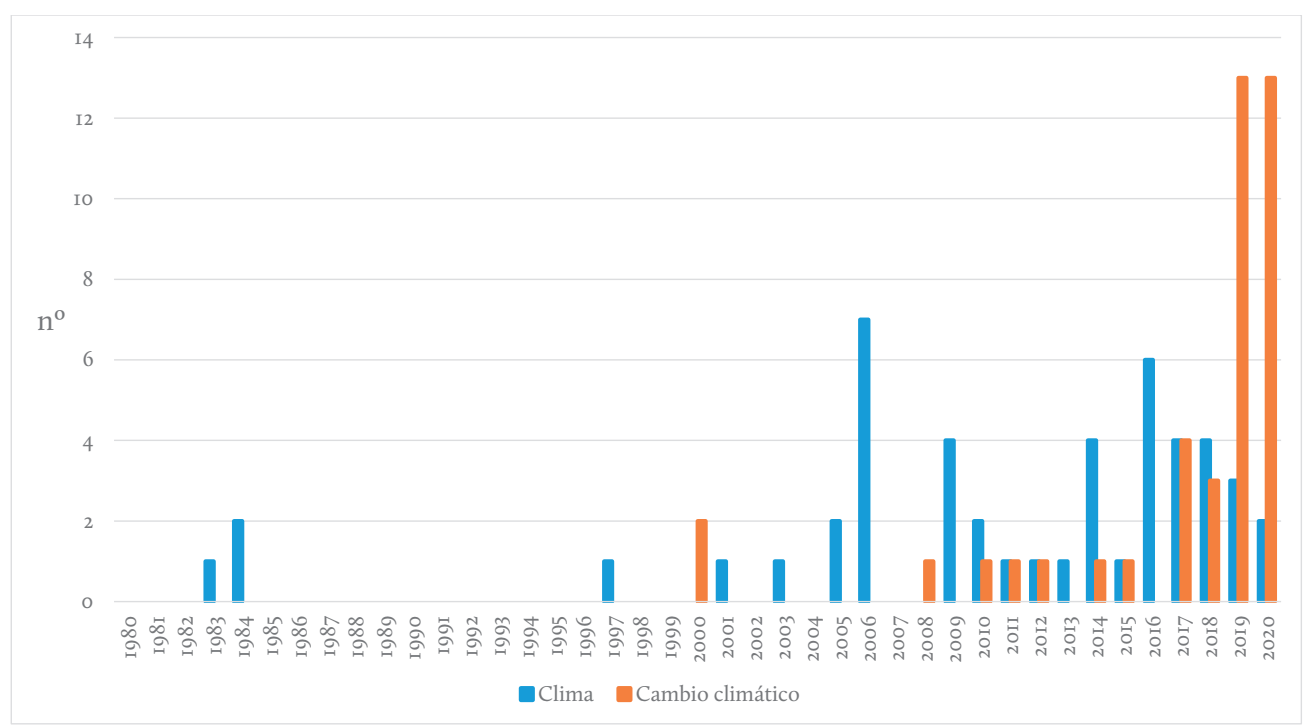

FIGURA 3. EVOLUCIÓN DE LAS PUBLICACIONES SOBRE DIDÁCTICA DEL CLIMA Y CAMBIO CLIMÁTICO (19802020). Elaboración propia

En vinculación con las contribuciones identificadas sobre el cambio climático, cabe señalar que algunas de ellas tratan trasversalmente sobre los riesgos naturales. A su vez, es conveniente destacar que dentro de estos ejes (clima y cambio climático) se pueden distinguir las publicaciones en función de su objeto de estudio: I) trabajos vinculados con la enseñanza (propuestas, experiencias, uso de recursos, análisis 
curricular, etc.); 2) investigaciones sobre el análisis de las representaciones sociales (alumnado y profesorado); y 3) publicaciones sobre el análisis de libros de texto.

Los trabajos sobre el Climatología han sido constantes, especialmente entre el periodo 2005-2016 registrando una ligera disminución en los últimos 4 años. En la década 200I-20ı ha influido notablemente la publicación del $n^{\circ} 8$ de la revista Didáctica Geográfica (año 2006) en la que 4 contribuciones versan sobre la enseñanza del clima. También, en la última década cabe destacar las publicaciones derivadas del VII Congreso Ibérico de Didáctica de la Geografía celebrado en Alicante (2015) con 4 trabajos. Respecto a las temáticas, de manera agrupada entre clima y cambio climático, la mayoría de los trabajos se relacionan con la enseñanza $(58,4 \%$; $\mathrm{n}=52$ ). En segundo lugar, destacan las publicaciones sobre el análisis de las representaciones sociales $(29,2 \% ; n=26)$ y finalmente, las vinculadas con el análisis de libros de texto ( $15,7 \% ; \mathrm{n}=\mathrm{I} 4)$. Si se tienen en cuenta los trabajos según temáticas y ejes (clima y cambio climático), se observa una diferencia notable, a saber: en las publicaciones sobre el clima destacan aquellas relacionadas con la enseñanza $(76,6 \% ; n=36)$, mientras que las de cambio climático el análisis de las representaciones sociales $(48,9 \% ; n=22)$ (ver Figura 4).

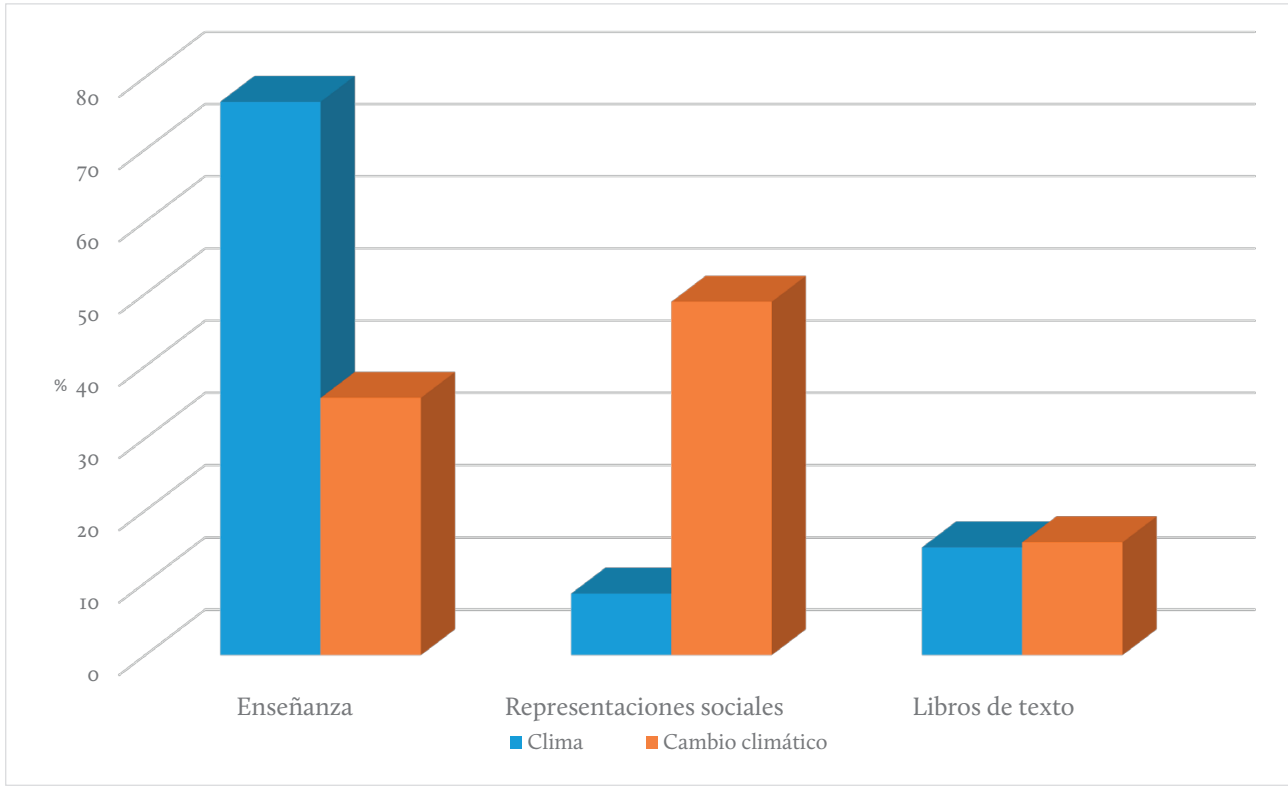

FIGURA 4. TEMÁTICAS OBJETO DE ESTUdIO DE DIDÁCTICA DE LA CLIMATOLOGíA (1980-2020). Elaboración propia

\subsection{LA ENSEÑANZA DE LA CLIMATOLOGÍA. EXPERIENCIAS Y PROPUESTAS DIDÁCTICAS}

Una vez analizados los resultados globales, a continuación, se procederá al análisis de las publicaciones en función de las temáticas y ejes identificados. La primera es la vinculada con la enseñanza que, como ya se ha comentado en el punto anterior, sus trabajos representan el $58,4 \%(n=52)$. Para el caso de las relacionadas con el clima, estas representan el $76,6 \%(n=36)$. Son estas las primeras publicaciones 
identificadas ya en la década de los ochenta con trabajos que tienen que ver con propuestas didácticas y experiencias en el aula (Clavero \& Raso, I983; Martín, I984; Repetto, I984) (ver Tabla I). Para el caso del trabajo de Clavero \& Raso (I983) este destaca por presentar dos partes diferenciadas (propuestas didácticas y una parte de actividades). Además, esta es la única publicación en formato libro que se ha identificado. En cuanto a la publicación de Martín (I984), esta trata sobre experiencias en el aula (EGB), mientras que la contribución de Repetto (I984) propone la realización en clase de diferentes actividades relacionadas con el estudio de elementos del clima (presión, temperatura, humedad atmosférica), fenómenos meteorológicos, predicción del tiempo y los condicionantes del clima canario.

En la década de los noventa tan sólo se ha identificado un trabajo (Navalón, I997), no siendo hasta la entrada del s. XXI (I3 trabajos en la década 200I-20I0) cuando se lleva a cabo una consolidación y tendencia ascendente de esta temática. Algunos de los principales exponentes de este periodo son los trabajos de Sánchez-Ogallar (2005a; 2005b), Marrón (2006), Ramiro (2006), De Pro \& Ezquerra (2006) y los de Cruz (2006; 2008; 2010). Por ejemplo, el trabajo de Ramiro (2006) destaca por ser una de las primeras experiencias didácticas que tratan sobre el fenómeno de la isla de calor urbana. El objetivo de este trabajo es constatar la existencia de este fenómeno en pequeñas localidades (25.00o habitantes). A partir del desarrollo de su experiencia en alumnos/as de Educación Secundaria (asignatura de Ciencias Sociales: Geografía e Historia), y su aprovechamiento didáctico, concluyó que, según los datos obtenidos, no se puede comprobar la evolución de este fenómeno con su formación y desaparición, en cambio, sí que constató la existencia e inicio. En el trabajo de Marrón (2006) se presenta una propuesta para enseñar y aprender los climas españoles a partir de los refranes, mientras que en la contribución de De Pro \& Ezquerra (2006), a partir del uso y propuestas didácticas de los espacios meteorológicos de televisión, los autores tienen el objetivo de estudiar los rasgos de estos espacios para, por una parte, explorar cómo pueden influir a la formación científica de la sociedad y, por otra, valorar cómo pueden ser aprovechados en el ámbito escolar. Otra de las ideas que cabe destacar de estos autores es que ya hace dos décadas ponían de manifiesto la complejidad del uso de estos recursos en las clases.

Los trabajos que finalizan esta década son los realizados por Cruz $(2006 ; 2008$; 20II). Para el caso de la contribución de 2006, esta destaca por ser una propuesta diseñada y realizada con alumnos/as del Programa de Diversificación Curricular. El objetivo es que el alumnado sea capaz de observar, examinar e interpretar mínimamente el funcionamiento de la atmósfera y sus consecuencias. Cruz (2006) argumenta que la observación del tiempo atmosférico sin aparatos puede ser una buena idea para enseñar Climatología, al igual que una actividad donde se fomenten aspectos didácticos en relación con la atención a la diversidad. Respecto a la publicación de 2008 , esta tiene el objetivo de presentar una propuesta para el análisis de tipos de nubes como recurso interdisciplinar (Geografía e Historia del Arte en Ciencias Sociales) con alumnado de Educación Secundaria. La secuencia de la actividad propuesta por Cruz (2008) tiene varias fases: I) se explica al alumnado los tipos de nubes y sus características; 2) se elabora un croquis interpretativo de 
los tipos del cielo (características y tipo de nubes; y 3 ) se analizan las nubes en las obras pictóricas escogidas, al igual que sus rasgos estilísticos.

En la última década 20II-2020 la tendencia de publicaciones sobre esta temática ha ido en aumento $(\mathrm{n}=\mathrm{I9})$ ). Algunos de estos trabajos son los realizados por Marrón (20II), Olcina (20I4), Martínez-Romera (2013), García de la Vega (20I6), MartínezIbarra et al. (2016), Martínez-Fernández \& Olcina (2019), Morales-Yago (2016), Morote \& Moltó (2017) y Sebastiá \& Tonda (20I8). En el trabajo de Marrón (20II), esta autora continúa con la línea de estudio de 2006 sobre la enseñanza del clima a partir de las fuentes orales en la que destaca que estas son una forma de sabiduría tradicional basadas en la experiencia y observación individual-colectiva de diversos fenómenos (naturales, sociales, económicos, etc.), siendo el resultado de análisis repetidos por la sociedad durante siglos, de ahí, el elevado nivel de veracidad. Marrón (20II) destaca que la mayoría de los refranes se cumplen, por tanto:

«refrán que no se cumple con una alta regularidad acaba desapareciendo («No hay refrán que no sea verdadero», «Cien refranes, cien verdades», «No hay refrán que no diga una verdad; y si una no, es porque dice dos», «Refrán no verdadero, poco duradero)» (p. 109).

Una mención especial merece los trabajos vinculados con el uso y propuesta de climogramas (Martínez-Romera, 2013; Corrales et al., 2018). Sin duda, es el recurso más utilizado y analizado en Didáctica de la Climatología, especialmente en las clases y libros de texto. No obstante, autores como Olcina (20I7) han señalado sus limitaciones y errores que se suelen cometer en los manuales escolares, especialmente, los problemas vinculados con la escala. También el trabajo de Martínez-Romera (2013) es uno de los primeros que tratan sobre la enseñanza del clima y las TIC. Este autor ya ponía de manifiesto que la adopción de la interactividad de los recursos web, en las aplicaciones disponibles para mejorar su aprendizaje, se encontraban en una fase inicial, dificultando su aprovechamiento para determinadas posibilidades inherentes a la tecnología (manipulación de datos, la simulación numérica, etc.).

Cabe mencionar el análisis que realiza Olcina (2014) sobre la contribución de Immanuel Kant como profesor de Geografía y, especialmente a la enseñanza de la Climatología. Otros trabajos como el de Morales-Yago (20I6) tiene que ver con los mapas de tiempo y el aprendizaje cooperativo, mientras que el de García de la Vega (20I6) trata sobre la revisión del currículo y rigor científico en los textos literarios vinculados con el estudio del clima. Asimismo, en relación con el análisis curricular, cabe destacar el estudio de Martínez-Fernández \& Olcina (2019) que, partiendo de una revisión y la valoración de los contenidos curriculares sobre el tiempo y clima que se enseñan en la educación obligatoria, plantean diferentes propuestas para mejorar la enseñanza-aprendizaje de la Climatología en la etapa escolar. Finalmente, cabe destacar que las últimas contribuciones identificadas tienen que ver con la propuesta y experiencias de recursos como son los centros de interpretación y museos sobre el clima, en este caso, el Museo del Clima de Beniarrés (Alicante) (Morote \& Moltó, 20I7; Moltó \& Morote, 2019; Moltó, 2019). 
TABLA 1. PUBLICACIONES SOBRE DIDÁCTICA DEL CLIMA Y EL CAMBIO CLIMÁTICO (TEMÁTICA «EXPERIENCIAS Y PROPUESTAS DIDÁCTICAS»)

\begin{tabular}{|c|c|}
\hline $\begin{array}{l}\text { CONTRIBUCIONES SOBRE } \\
\text { DIDÁCTICA DEL CLIMA }\end{array}$ & $\begin{array}{l}\text { CONTRIBUCIONES SOBRE DIDÁCTICA } \\
\text { DEL CAMBIO CLIMÁTICO }\end{array}$ \\
\hline $2011-2020$ & $2011-2020$ \\
\hline $\begin{array}{l}\text {-Moltó (2019) } \\
\text {-Moltó \& Morote (2019) } \\
\text {-Abarca et al. (2018) } \\
\text {-Corrales et al. (2018) } \\
\text {-González \& Arias (2018) } \\
\text {-Sebastiá \& Tonda (2018) } \\
\text {-Campo \& Martínez (2017) } \\
\text {-Martínez-Fernández \& Olcina (2019) } \\
\text {-Morote \& Moltó (2017) } \\
\text {-García de la Vega (2016) } \\
\text {-Martínez et al. (2016) } \\
\text {-Morales-Yago (2016) } \\
\text {-Morote (2016) } \\
\text {-Méndez y Martínez-Martínez (2015) } \\
\text {-Grasso (2014) } \\
\text {-Olcina (2014) } \\
\text {-Martínez-Romera (2013) } \\
\text {-Genovart (2012) } \\
\text {-Marrón (2011) }\end{array}$ & $\begin{array}{l}\text {-Moreno-Vera (2020) } \\
\text {-Morote \& Hernández (2020a) } \\
\text {-González et al. (2020) } \\
\text {-Caride \& Meira (2019) } \\
\text {-Gómez \& Corrochano (2019) } \\
\text {-Morote (2019a) } \\
\text {-Ormaetxea et al. (2019) } \\
\text {-Sánchez-Emeterio (2019) } \\
\text {-Ouriachi et al. (2017a) } \\
\text {-Ouriachi et al. (2017b) } \\
\text {-Doménech-Casal (2014) }\end{array}$ \\
\hline 2001-2010 & 2001-2010 \\
\hline $\begin{array}{l}\text {-Cruz (2010) } \\
\text {-Romero (2020) } \\
\text {-Cruz (2008) } \\
\text {-Cruz (2006) } \\
\text {-De Pro \& Ezquerra (2006) } \\
\text {-López-Martín (2006) } \\
\text {-Marrón (2006) } \\
\text {-Ramiro (2006) } \\
\text {-Rodrigo (2006) } \\
\text {-Sánchez-Ogallar (2005a) } \\
\text {-Sánchez-Ogallar (2005b) } \\
\text {-Tonda \& Sebastiá (2003) } \\
\text {-Juan \& Molina (2001) }\end{array}$ & $\begin{array}{l}\text {-Gómez et al. (2010) } \\
\text {-González \& Meira (2009) } \\
\text {-Soñora et al. (2009) }\end{array}$ \\
\hline 1991-2000 & 1991-2000 \\
\hline -Navalón (1997) & $\begin{array}{l}\text {-Olcina \& Zamora (2000) } \\
\text {-Sánchez-Ogallar (2000) }\end{array}$ \\
\hline $1980-1990$ & $1980-1990$ \\
\hline $\begin{array}{l}\text {-Martín (1984) } \\
\text {-Repetto (1984) } \\
\text {-Clavero \& Raso (1983) }\end{array}$ & - \\
\hline
\end{tabular}

Elaboración propia 
En relación con los trabajos sobre el cambio climático, no es hasta la década 2OII-2020 cuando se puede hablar de una consolidación de esta temática de estudio ya que, 11 contribuciones sobre un total de i6 (el 68,8\%) se han publicado en este periodo. Cabe destacar que las dos primeras investigaciones identificadas son las de Olcina \& Zamora (2000) y Sánchez-Ogallar (2000). El primero trata sobre el cambio climático y sus efectos asociados (riesgos naturales) a través de los recursos didácticos disponibles en Internet. En cuanto a la segunda publicación, SánchezOgallar (2000) explica la complejidad de la trasposición didáctica a la hora de enseñar el clima. En este trabajo se argumenta que el estudio en el aula sobre las causas y efectos de este fenómeno presenta una gran complejidad didáctica, por tratarse de un tema multicausal que exige una programación sistemática y el despliegue de estrategias metodológicas adecuadas. Este autor diferencia dos grupos de medidas: I) el clima como realidad (la explicación y la descripción); y 2) el clima como problema (el análisis y la valoración). Sánchez-Ogallar (2000) ya ponía de manifiesto hace dos décadas que era importante que el sistema educativo insertara a medio plazo en sus proyectos curriculares una visión del clima basada en «el Clima como Problema» para que estas medidas tuvieran eficacia.

Un segundo grupo de trabajos son aquellos que se han publicado en el equipo SEPA (Universidad de Santiago de Compostela) (Caride \& Meira, 2019; Soñora, Rodríguez \& Trotiño, 2009). También cabe destacar algunos trabajos que tratan el cambio climático y sus riesgos asociados (ver Morote \& Hernández, 2020). El objetivo de este último es examinar el rendimiento académico de los/as alumnos/as del Máster en Planificación y Gestión de Riesgos Naturales (Universidad de Alicante) (cursos 20I7-20I8 y 20I8-20I9). Los autores han analizado que el progreso académico ha sido el que se esperaba y que los diversos casos de estudio que se exponen en clase han permitido mejorar las creencias del estudiantado sobre diferentes estereotipos sobre este fenómeno y sus consecuencias.

\subsection{ANÁLISIS DE REPRESENTACIONES SOCIALES. LA IMPORTANCIA DE CONOCER QUÉ ES LO QUE SABE EL ALUMNADO Y EL PROFESORADO}

La segunda temática analizada ha sido aquella que se vincula con las representaciones sociales. En esta se han identificado un total de 26 trabajos siendo la mayoría (el 84,6\%; n=22) los que se vinculan con el cambio climático. En primer lugar, cabe explicar qué son las representaciones sociales. Según Moscovici (I978), la teoría de las representaciones sociales aparece como paradigma que permite explicar el funcionamiento del canon escolar en la formación en Ciencias Sociales. El intento de mejorar el aprendizaje de las Ciencias Sociales se enfrenta a las concepciones espontáneas que posee el alumnado, docentes, editores de manuales escolares y legisladores, pues se han generado en un contexto histórico dominado por una cultura hegemónica que influye en todos los agentes escolares (Borg, 2003; Jodelet, I994). También es importante subrayar que las representaciones sociales permiten comprender las particularidades y efectos de 
la inserción de los individuos en contextos culturales específicos y los procesos de incorporación de los significados hegemónicos del mundo social que hacen.

TABLA 2. PUBLICACIONES SOBRE DIDÁCTICA DEL CLIMA Y EL CAMBIO

CLIMÁTICO (TEMÁTICA «ANÁLISIS DE REPRESENTACIONES SOCIALES»)

\begin{tabular}{|c|c|}
\hline $\begin{array}{l}\text { CONTRIBUCIONES SOBRE } \\
\text { DIDÁCTICA DEL CLIMA }\end{array}$ & $\begin{array}{l}\text { CONTRIBUCIONES SOBRE DIDÁCTICA } \\
\text { DEL CAMBIO CLIMÁTICO }\end{array}$ \\
\hline $2011-2020$ & $2011-2020$ \\
\hline $\begin{array}{l}\text {-Campo \& García (2020) } \\
\text {-Rubio \& Martínez-Medina (2014) }\end{array}$ & $\begin{array}{l}\text {-García-Vinuesa et al. (2020) } \\
\text {-Morote (2020c) } \\
\text {-Morote \& Hernández (2020b) } \\
\text {-Morote \& Souto (2020) } \\
\text {-Robredo \& Ladreda (2020) } \\
\text {-Varela et al. (2020) } \\
\text {-Velasco et al. (2020) } \\
\text { - - Ferrari et al. (2019) } \\
\text {-García-Vinuesa et al. (2019) } \\
\text {-García et al. (2019) } \\
\text {-López-Guerrero (2019) } \\
\text {-Morote (2019b) } \\
\text {-Morote et al. (2019) } \\
\text {-Roldán et al. (2019) } \\
\text {-Meira et al. (2018) } \\
\text {-Roldán et al. (2018) } \\
\text {-Sanchis et al. (2018) } \\
\text {-Benavides et al. (2017) } \\
\text {-García-Rodeja \& De Oliveira (2012) } \\
\text {-Punter et al. (2011) }\end{array}$ \\
\hline 2001-2010 & 2001-2010 \\
\hline $\begin{array}{l}\text {-Tonda \& Sebastiá (2003) } \\
\text {-Valbuena \& Valverde (2006) }\end{array}$ & $\begin{array}{l}\text {-Arto (2009a) } \\
\text {-Arto (2009b) }\end{array}$ \\
\hline
\end{tabular}

Elaboración propia. Nota: en la temática de análisis de representaciones sociales no se han identificado trabajos en las décadas de 1980-1990 y 1991-2000.

Para el caso de los estudios sobre el clima, tan sólo se han identificado 4 contribuciones (ver Tabla 2). En el trabajo de Tonda \& Sebastiá (2003) (que también es una contribución de propuesta didáctica), es uno de los primeros estudios desde la Didáctica de la Climatología en analizar la representación social de los/ as estudiantes. A partir del diseño de una encuesta, los autores escogieron el clima como un elemento cotidiano del alumnado y presente en el currículo. Los datos que obtuvieron corroboran diferentes carencias vinculadas con sus hipótesis iniciales. Algunas de las conclusiones de Tonda \& Sebastiá (2003) son que el alumnado: I) no comprende la utilidad de la proporcionalidad; 2) no sabe realizar mediciones y ofrecer los resultados de las mismas; 3) no reconoce la importancia de la expresión visual 
(colores); y 4) no valora la estandarización o consideración de unos referentes como una herramienta que posibilita la comparación.

Por su parte, en el trabajo de Valbuena \& Valverde (2006) los autores plantean la necesidad de desarrollar ejemplos prácticos con alumnado universitario en asignaturas no obligatorias, permitiendo, de esta manera, proponer herramientas de observación a partir del territorio y de la sociedad, para así resolver las carencias. Algunas de las conclusiones a las que estos autores han llegado son que, la acumulación de datos sobre el tiempo y clima, a partir de la observación y la experimentación, permite corroborar algunos de los rasgos generales del clima que frecuentemente se ve influenciado por las diferencias que a nivel local introduce la vegetación, el relieve o incluso las formas de vida de sus habitantes. Otros trabajos como el de Rubio \& Martínez (20I4) tiene el objetivo de analizar si los/as estudiantes del Grado de Educación Primaria son competentes en el conocimiento de la Climatología. Las conclusiones extraídas de esta contribución son que el alumnado suele conocer las principales diferencias entre tiempo y clima, pero no controlan de una manera eficaz las unidades de medida y las magnitudes físicas. También, estos autores han comprobado que el alumnado no reconoce correctamente los diversos climas a partir de una amalgama de datos y factores. Finalmente, Rubio \& Martínez (2014) concluyen que el alumnado tiene parte de los conocimientos adquiridos durante su formación, sin embargo, son incapaces de utilizar los recursos para comprender el funcionamiento del clima.

Respecto a los trabajos sobre las representaciones sociales del cambio climático, resulta de vital importancia examinar estas cuestiones para poder analizar lo que piensan y perciben sobre este fenómeno, tanto el alumnado como los docentes. Unos de los trabajos precedentes sobre esta temática son los de Arto (2009a; 2009b). Arto (2009b), a partir de una muestra de 636 casos, ha analizado la relación en las respuestas de este fenómeno con otras cuestiones ambientales, concretamente el deterioro de la capa de ozono, siendo citado en un 25,4\% de los casos. También Arto (2009b), a partir del uso del dibujo y texto libre, ha tratado el cambio climático desde una visión más informal, además de favorecer que el discurso del alumnado que ha participado se plasmase en un producto comprensivo que permitiese dar forma a «objetos» -primero el «cambio climático» y después «el deterioro de la capa de ozono»- que implican un alto grado de complejidad.

Otro trabajo como el de Morote, Campo \& Colomer (2019) tiene la finalidad de analizar de dónde los docentes en formación (Educación Primaria) reciben la información sobre el cambio climático. Los datos indican que los principales medios son internet y la televisión. La cifra asciende al 86,2\% desagregándose entre internet (32,8\%), TV (31,3\%) y redes sociales (Facebook, twitter, etc.) (22,1\%). También cabe indicar el reducido peso que tiene la información recibida que procede del ámbito académico y científico (desde la universidad) con tan sólo el 5,3\%. Como explican los autores, con estos datos se pueden deducir los posibles estereotipos e información falsa que pueden recibir (las llamadas fake news) sobre el cambio climático. También destacan aquellos trabajos sobre los efectos de este fenómeno como son las inundaciones. Respecto a estas contribuciones cabe destacar los estudios de Morote y Souto (2020) y Morote \& Hernández (2020). En cuanto al primero, los autores han analizado como la mayoría del futuro profesorado no ha 
recibido formación sobre esta temática (sólo el I2,I\% recibió formación), mientras que en el segundo, los autores han analizado la escasa importancia que le dan a estos contenidos para ser tratados en la Educación Primaria. Llamativo es el resultado obtenido de aquellos que sí recibieron formación, que coinciden con los que le dan una menor importancia (2,7 sobre 5 ).

\subsection{CLIMATOLOGÍA Y LIBROS DE TEXTO. ANÁLISIS Y PROPUESTAS DE MEJORA}

La tercera temática que se ha identificado es aquella que tiene que ver con las publicaciones que examinan los manuales escolares. Esta línea de estudio es la más actual (la mayoría de los trabajos pertenecen a la década de 20II-2020) y la que representa un número menor de contribuciones (ver Figura 5). El número total de trabajos asciende a I4 (el I3,5\% sobre el total), distribuyéndose equitativamente entre publicaciones sobre Didáctica del clima y cambio climático. Entre los primeros cabe destacar cronológicamente las contribuciones de Rodrigo (2006), Ramos \& Calonge (20I4), Martínez-Medina \& López-Fernández (2016), Martínez-Medina (20I6), Olcina (2017) o Martínez-Fernández \& Olcina (2019).

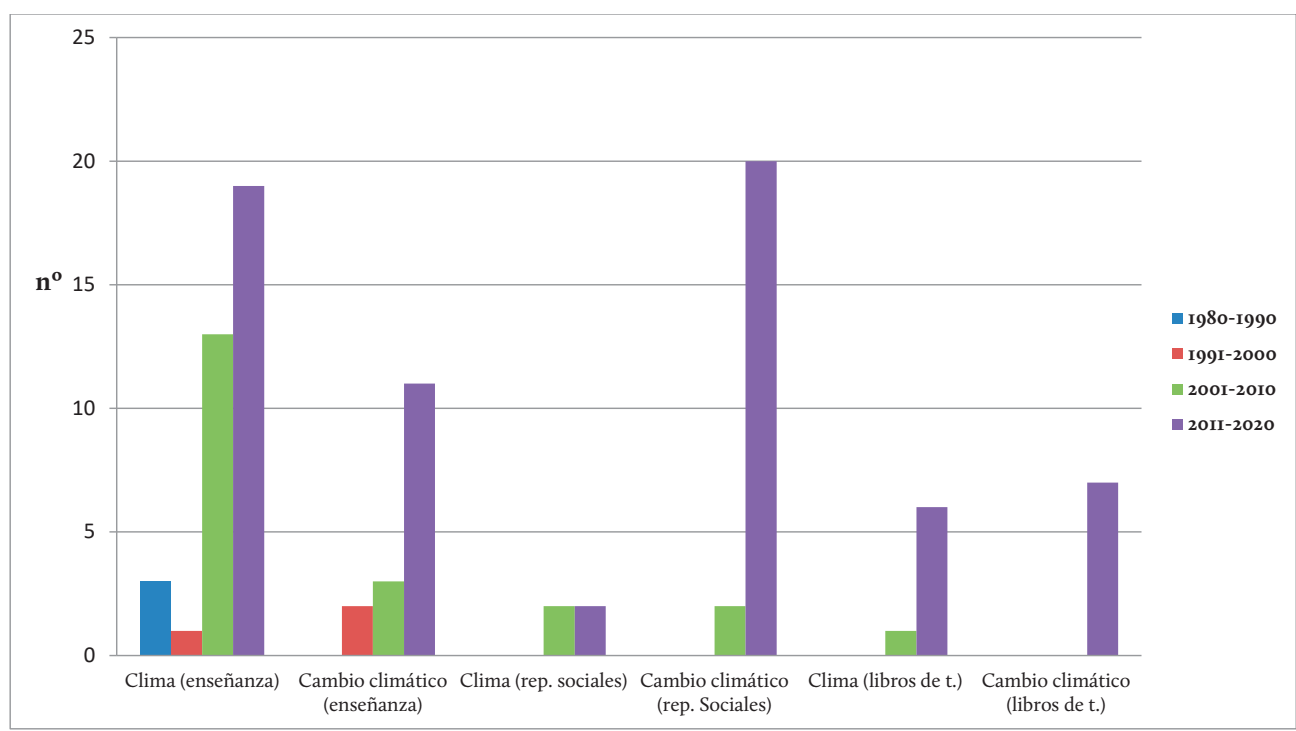

FIGURA 5. EVOLUCIÓN DE LOS TRABAJOS DE DIDÁCTICA DE LA CLIMATOLOGÍA SEGÚN TEMÁTICAS Y EJES (CLIMA Y CAMBIO CLIMÁTICO) (1980-2020). Elaboración propia

El primer referente es el trabajo de Rodrigo (2006), de la Universidade Estadual Paulista (Brasil), también considerado como uno de los primeros autores no españoles en publicar un trabajo sobre Didáctica de la Climatología en vinculación con el contexto español. Rodrigo (2006) ha analizado el lenguaje de la cartografía del clima de diferentes manuales escolares de la ESO de España y Brasil. Algunos de los resultados obtenidos son las deficiencias relacionadas con la no presencia de la escala, la proyección, la orientación y la leyenda. Para el caso del trabajo de Ramos \& Calonge (2014), estos autores se hacen las siguientes preguntas: 
«1) ¿hasta qué punto ha tenido lugar una mejora en la calidad del currículo de Geografía Física que se presenta en estos libros?; y 2) ¿es coherente el análisis de los climas de España con el nivel académico al que se dirigen los libros de texto?» (p. 129).

Ramos \& Calonge (20I4), para responder y resolver estas cuestiones, tras su análisis, llegan a la conclusión de que en los manuales, en la explicación de los climas se elaboran notables desajustes entre las propuestas metodológicas y los contenidos que se insertan en los libros. Ramos \& Calonge (2014) apuestan por la renovación de los libros de texto (parte de la Climatología), a fin de clarificar el currículo a partir de:

«1) unos conceptos climatológicos expresados con un sentido geográfico; 2) una adecuación de las clasificaciones al nivel académico ( $2^{\circ}$ de Bachillerato) al que van dirigidos los libros, de tal manera que los climas de España resulten fácilmente comprensibles en términos generales sin necesidad de complejas subdivisiones; y 3) a través de unos mapas temáticos que identifiquen con claridad la localización y la extensión de los dominios climáticos españoles» (p. 149).

También cabe destacar las contribuciones que han sido precedentes y de guía (Martínez-Medina, 20ı6; Martínez-Medina \& López-Fernández, 20I6) para los trabajos que han tratado los manuales a partir del análisis cuantitativo de los recursos, actividades y las imágenes. Los trabajos de Martínez-Medina (2016) y Martínez-Medina \& López-Fernández (2016) han analizado la explicación de la Climatología en los manuales de Educación Primaria (Ciencias Sociales). Según los resultados obtenidos por Martínez-Medina (2016), el análisis del tiempo y clima es poco esperanzador ya que no existe un criterio común que enseñe los factores y elementos del clima. Este autor explica que esta cuestión se repite en la clasificación de los climas de España y, además, es diferente en todos los libros analizados, incluso manuales de una misma editorial. Para el caso de los climogramas, Martínez-Medina (20I6) pone de manifiesto que resulta llamativo las diferencias en el tratamiento de estos recursos ya que en casi todos se pide su interpretación, en otros además su elaboración, pero en la mayoría de los ejemplos son actividades que están totalmente desvinculadas con la enseñanza de los climas.

A estas mismas conclusiones también ha llegado Olcina (2017) (manuales escolares de Educación Secundaria) en la que explica que las escalas de los climogramas dan en ocasiones impresiones engañosas cuando se comparan dos localidades. De ahí la propuesta de elaborar diagramas con idéntica escala (térmica y pluviométrica) a la hora de caracterizar y comparar variedades climáticas. También Olcina (20I7) explica algunas cuestiones positivas tras su análisis que se reflejan en los libros de texto. Por ejemplo, para los manuales de $3^{\circ}$ de ESO: I) se compara el mapa de climas del mundo con el mapa de poblamiento mundial; 2) relación entre climas y paisajes naturales; 3) se incluye cartografía con la extensión de la capa de hielo (épocas glaciares; Cuaternario) para reflejar que el clima de la Tierra siempre ha cambiado. Esta reflexión es interesante a la hora de situar el proceso actual de calentamiento térmico planetario, en un contexto más general de la evolución del clima a lo largo de la historia; 4) relación entre precipitaciones en España y recursos hídricos 
en los embalses; 5) apartado de riesgos naturales y relación con la población; y 6) imágenes con efectos de fenómenos extremos (tsunamis) (aunque como bien apunta el autor, no son riesgos climáticos). Otra publicación, la de Morote (2020a), tiene el objetivo de examinar los recursos y actividades sobre Climatología (libros de texto de Educación Primaria). Los resultados a los que ha llegado este autor son, respecto a los recursos, que la mayoría tienen que ver con fotografías y, en vinculación con las actividades, la mayoría se vinculan con la parte principal de la unidad didáctica. En relación con el uso de las ilustraciones, este autor ha comprobado que generalmente se presentan como figuras de apoyo.

TABLA 3. PUBLICACIONES SOBRE DIDÁCTICA DEL CLIMA Y EL CAMBIO CLIMÁTICO (TEMÁTICA «ANÁLISIS DE LIBROS DE TEXTO»)

\begin{tabular}{|l|l|}
\hline $\begin{array}{l}\text { CONTRIBUCIONES SOBRE } \\
\text { DIDÁCTICA DEL CLIMA }\end{array}$ & $\begin{array}{l}\text { CONTRIBUCIONES SOBRE DIDÁCTICA } \\
\text { DEL CAMBIO CLIMÁTICO }\end{array}$ \\
\hline $\mathbf{2 0 1 \mathbf { 1 } - 2 0 2 0}$ & $\mathbf{2 0 1 \mathbf { 1 } - 2 0 2 0}$ \\
\hline -Morote (2020a) & -Morote (2020b) \\
-Martínez-Fernández y Olcina (2019) & - Morote \& Olcina (2020) \\
-Olcina (2017) & -Navarro et al. (2020) \\
-Martínez-Medina (2016) & -Morote (2019C) \\
-Martínez-Medina \& López-Fernández (2016) & -Arrebola \& Martínez (2017) \\
-Ramos \& Calonge (2014) & -Olcina (2017) \\
\hline 2001-2010 & -Serantes (2015) \\
\hline -Rodrigo (2006) & $\mathbf{2 0 0 1 - 2 0 1 0}$ \\
\hline
\end{tabular}

ELABORACIÓN PROPIA. NOTA: EN LA TEMÁTICA SOBRE EL ANÁLISIS DE LIBROS dE TEXTO NO SE HAN IDENTIFICADO TRABAJOS EN LAS DÉCADAS DE 1980-1990 Y 1991-2000

En cuanto a las contribuciones sobre el cambio climático, cabe destacar, por ejemplo, las de Serantes (2015), Arrebola \& Martínez (2017), Morote \& Olcina (2020) y Navarro, Moreno \& Rivero (2020). Aquí, también cabe resaltar algunas ideas expresadas por Olcina (20I7) sobre diferentes cuestiones de los contenidos que tienen que ver con el cambio climático. Por ejemplo, este autor explica que es interesante la incorporación de contenidos que complementan aspectos nuevos como los riesgos atmosféricos o el mismo cambio climático. Pero, como explica este autor, debe escogerse bien el enfoque a enseñar estas temáticas porque no resulta positivo tratarlos con un excesivo catastrofismo. Estos temas deben enseñarse con realismo: mostrando lo que realmente conllevan los riesgos y el cambio climático. A estas mismas conclusiones han llegado Morote \& Olcina (2020) en vinculación con los libros de texto de Educación Primaria (Ciencias Sociales). Estos autores destacan 3 conclusiones que se deberían corregir a la hora de la edición de estos recursos: I) el cambio climático se explica con muy poco rigor científico, donde son presentes las explicaciones no corroboradas con fuentes o datos científicos; 2) no hay presencia de la vulnerabilidad humana como factor que aumenta el riesgo. Al respecto, en ningún manual se cita este factor, siendo la peligrosidad (el «cambio del clima») la causa principal de los desastres; y 3 ) la excesiva presencia de ilustraciones catastrofistas. 


\section{DISCUSIÓN}

Con este trabajo se ha comprobado el estado de la cuestión y tendencia de las publicaciones relacionadas con la Didáctica de la Climatología temática cada vez con un mayor interés para el ámbito educativo por las implicaciones sociales y de concienciación en las generaciones presentes y futuras. En relación con las hipótesis de partida, estas se han cumplido. Se ha podido comprobar que la evolución general del número de contribuciones ha sido ascendente, especialmente en la última década. Por ejemplo, si hasta 2010 el total de trabajos ascendía a 24, en los últimos diez años las publicaciones han sido 65 (el 73,0\%). Se ha identificado un total de 89 trabajos, un número que pone también de manifiesto el interés de los/ as investigadores/as que trabajan en Didáctica de la Climatología. En este sentido, cabe recordar que hasta la fecha de la publicación del trabajo de Tonda \& Sebastiá (2003) tan sólo había publicadas una decena de investigaciones. Respecto al cambio climático se ha podido comprobar que se ha convertido recientemente en el tema «estrella» en todas las temáticas. También se ha constatado que durante el s. XXI diferentes eventos y números especiales de revistas han podido influir en el aumento de las contribuciones sobre la enseñanza de la Didáctica de la Climatología: VII Congreso Ibérico de Didáctica de la Geografía (2015), y el nº8 de la revista de Didáctica Geográfica (2006).

En cuanto a las temáticas identificadas se han categorizado los trabajos en tres: I) enseñanza (experiencias, propuestas y recursos); 2) análisis de representaciones sociales; y 3 ) análisis de libros de texto. Cabe indicar que tan sólo la vinculada con la primera ha estado presente en las 4 décadas. Respecto a los/as autores/as, de manera general no se aprecia una línea continuista durante todo el intervalo de análisis (1980-2020). Destacan en momentos puntuales autores/as que le han dedicado una especial atención, lo que pone de manifiesto que los investigadores/as-docentes no se dedican exclusivamente a esta línea de trabajo. Tan sólo 4 autores tienen 3 o más contribuciones: Cruz ( $\mathrm{n}=3)$; Sánchez-Ogallar $(\mathrm{n}=3)$; Moltó $(\mathrm{n}=3)$; Olcina $(\mathrm{n}=5)$; Morote $(\mathrm{n}=\mathrm{I} 3)$. De estos, las contribuciones de los tres últimos destacan porque se han concentrado en los últimos años y especialmente por ser publicaciones que tienen como contexto territorial la región valenciana, unas de las áreas más vulnerables a los efectos del cambio climático a nivel mundial (IPCC, 202I).

En relación con los trabajos sobre la enseñanza de la Climatología se ha podido comprobar las carencias, experiencias y propuestas didácticas para mejorar la explicación de estos contenidos. Tonda \& Sebastiá (2003), por ejemplo, dan importancia en iniciar desde la Educación Infantil la enseñanza sobre este tema. Por su parte, Valbuena \& Valverde (2006) ponen de manifiesto determinados problemas que surgen cuando en un territorio concreto no se dispone de estaciones pluvio-termométricas. Ante esta limitación, estos autores proponen acudir a otras fuentes que permitan acceder a información diversa y practicar observaciones y experiencias climáticas.

En relación al cambio climático, a nivel internacional numerosos trabajos indican el interés por enseñar este fenómeno en la etapa escolar (Carman et al., 2020; Namdar, 20I8), por las implicaciones que tiene como medida de concienciación y 
adaptación como explican, por ejemplo, para el caso brasileño Da Silva et al. (2019). Para llevar a cabo estas explicaciones se necesitan estrategias de aprendizaje eficaces (Muis \& Duffy, 2013), que tengan en cuenta el entorno local (Morote \& Olcina, 202Ib) y el uso de propuestas (Tucker \& Sherwood, 2020) y recursos que motiven al alumnado (Yow, 2014). En cuanto al entorno local, Morote \& Olcina (202Ib) plantean las propuestas denominadas «IOL» (que tengan en cuenta «Imaginación + Originalidad + Lo local»). Por su parte, Yow (20I4) propone enseñar Climatología y el cambio climático a partir de la cinematografía. Esto, para el caso español, también lo ha tenido en cuenta Moreno-Vera (2020) a partir de la película Wall-e.

En cuanto a la temática sobre las representaciones sociales, esta ha cobrado un protagonismo especial recientemente debido al interés de analizar las concepciones del alumnado y profesorado sobre el cambio climático. Trabajos como los de Morote et al. (2019) han comprobado como la mayoría de la información que recibe el profesorado en formación (Educación Primaria) sobre este fenómeno proviene de la TV, Internet y redes sociales, destacando el riesgo que puede suponer que esta información la tomen como propia. En la escala internacional destacan trabajos sobre las representaciones sociales del alumnado en la etapa escolar (Aksit et al., 2018). En Asia, por ejemplo, cabe citar los vinculados con el alumnado de Educación Secundaria (Chang \& Pascua, 20I6) o en México, en estudiantes universitarios (González \& Maldonado, 20I4). También, las representaciones sociales se han analizado en el profesorado (Hannah \& Rhubart, 2020; Wise, 20IO), e incluso entre comparaciones entre estados como ha realizado Khalidi \& Ramsey (2020) entre California y Texas (EE.UU.). Por su parte, Wise (2010) (investigación realizada en Colorado, EE.UU.), ha detectado ciertos conceptos erróneos sobre el cambio climático que están muy extendidos entre el profesorado (creencia de las dos causas principales -factores humanos y naturales-).

La tercera temática identificada (manuales escolares) es la que menor atención ha tenido y la que ha emergido en la última década. Como han resaltado Morote \& Olcina (2020) y Pagès (2008), el análisis de estos recursos es una línea de trabajo clásica en Didáctica de las Ciencias Sociales. No obstante, en España, esta línea de trabajo no está tan afianzada en Geografía como sí ha sucedido con Historia (Morote, 2020b). Ello se debe por el escaso número de geógrafos/as que se han dedicado a esta línea de investigación como ya manifestó Souto (2000) y Olcina (2017) y, recientemente Morote (2020b). El interés por analizar los manuales escolares en Ciencias Sociales es porque actualmente son el recurso principal utilizado en las aulas (Bel \& Colomer, 20I8) a pesar de que en los últimos años su uso ha descendido (Morote, 202ob). En relación con la Climatología, Martínez-Fernández \& Olcina (2019) indican que, en la actualidad, estos recursos son la herramienta didáctica más utilizada por el profesorado para enseñar esta temática.

Morote \& Olcina (2020) argumentan que la escasez de estudios sobre manuales escolares de Geografía aún empeora más para el caso de la revisión de los contenidos vinculados con el cambio climático (también por ser un tema reciente). En este sentido, Olcina (20I7) denuncia la escasez de estudios desde el análisis de estos recursos y la necesidad de consolidar esta línea de trabajo en la Geografía española. En el ámbito internacional, Bozkurt (2019) ha examinado la explicación de los 
contenidos sobre el clima en los libros de texto de Geografía (Turquía, Educación Secundaria). Este autor indica que los manuales presentan contenidos erróneos y, además, se caracterizan por presentar estereotipos sobre el clima. Esto mismo, también argumentan Morote \& Olcina (2020) en relación con los contenidos sobre este fenómeno en los libros de texto. Además, Martínez-Medina \& López-Fernández (20I6) explican que estos recursos reducen enormemente la accesibilidad a la información y documentación gráfica-cartográfica con la que se podría mejorar la explicación de la Climatología.

\section{CONCLUSIONES}

Con esta investigación se ha cubierto el vacío científico sobre el estado de la cuestión de los trabajos sobre la Didáctica de la Climatología en España en las 4 últimas décadas. Se ha podido comprobar que la producción científica (un total de 89 publicaciones) se ha incrementado, especialmente a partir del s. XXI, siendo el eje de mayor interés en los últimos años la temática del cambio climático. Respecto a esto último destacan las contribuciones sobre las representaciones sociales en el ámbito escolar y, en la última década, los análisis vinculados con los libros de texto. Además, esta investigación puede servir como apoyo bibliográfico para aquellos/as autores/as que deseen realizar o se inicien en esta línea de estudio.

El cambio climático es uno de los principales desafíos al que se enfrenta la sociedad del s. XXI. Esto mismo se ha visto reflejado en las investigaciones identificadas para el caso del ámbito educativo como aquí se ha constatado. En ello también ha podido influir el interés mostrado en los últimos años por las esferas políticas para mejorar la enseñanza de este fenómeno (Cumbre Cambio Climático de Madrid, 20I9 o la Ley de Cambio Climático -mayo de 202I-).

Las temáticas identificadas tienen un sentido lógico y de interés por parte de los/as autores/as. Por un lado, como investigadores/as-docentes, interesa realizar un diagnóstico del estado actual de las representaciones sociales del alumnado, futuro y actual profesorado, etc. Por otro, interesa conocer cómo se explican estos contenidos en los libros de texto. No cabe olvidar que en las clases de Ciencias Sociales y/o Geografía los manuales escolares siguen siendo el principal recurso utilizado. Una vez hecho este diagnóstico e identificado las carencias y aspectos positivos, es cuando se pueden establecer propuestas y presentar experiencias educativas a la comunidad docente.

Uno de los retos de investigación futura que se establece es analizar la evolución de la producción científica sobre esta temática en la presente década (202I-2030) y ello, por varias cuestiones: I) diferentes congresos que se han realizado en 202I en el que el cambio climático y el clima tiene una dedicación especial (I Congreso Internacional y XIII Nacional de Didáctica de la Geografía de la AGE celebrado en Girona) (4 contribuciones en la línea Temática I «La integración del desarrollo sostenible y de la educación para la sostenibilidad en la educación geográfica» y Temática 2 «El cambio climático y los objetivos de desarrollo sostenible»); y 2) la influencia que puede tener la Agenda 2030 (ODS) en la producción científica sobre 
las cuestiones del clima en el contexto español. También, será interesante comprobar si en los próximos años continúa la línea de estudio de determinados autores que han publicado en la última década. Otro reto de investigación será realizar un estudio bibliométrico sobre los trabajos ya identificados atendiendo a: I) la tipología de las contribuciones; 2) el idioma de la publicación; 3) la nacionalidad de los/as autores/as; 4) la internacionalización de los trabajos y 5) la etapa escolar a la que hacen referencia. Y como ya se ha comentado, actualizar esta base de datos para la década 202I-2030. Como limitación de trabajo cabe destacar la dificultad a la hora de localizar los trabajos que tienen que ver con actas de congresos o capítulos de libro que, en ocasiones, no aparecen en las bases de datos. Esto, para el caso de la Didáctica de la Geografía es un rasgo que la caracteriza ya que como ha comprobado Morote (2020b) en relación con los estudios sobre los libros de texto, el 44\% de las contribuciones se adscriben a esta tipología.

Finalmente, a modo de reflexión final, cabe indicar que la enseñanza del cambio climático presenta varios desafíos. El primero es lograr concienciar, no sólo al alumnado, sino también a la población en general del riesgo de tomar como propios los contenidos que se difunden desde la mayoría de los medios de información. Y, en segundo lugar, mejorar la formación del profesorado y alumnado escolar en estos contenidos para que de esta manera logren tener un espíritu crítico hacia estos temas. Sólo de esta manera se podrá conseguir una sociedad más resiliente y concienciada sobre este fenómeno global. 


\section{REFERENCIAS}

Ahmad, S. \& Numan, S.M. (2015). Potentiality of disaster management education through open and distance learning system in Bangladesh Open University. Turkish Online Journal of Distance Education, I6 (I), 249-260. https://doi.org/Io.I77I8/tojde.24I6I

Aksit, O., McNeal, K. S., Gold, A. U., Libarkin, J. C., \& Harris, S. (20i8). The influence of instruction, prior knowledge, and values on climate change risk perception among undergraduates. Journal of Research in Science Teaching, 55(4), 550-572. https://doi. org/IO.IO02/tea.2I430

Anyanwu, R. \& Grange, L.L. (20I7). The influence of teacher variables on climate change science literacy of Geography teachers in the Western Cape, South Africa. International Research in Geographical and Environmental Education, 26 (3), I93-206. https://doi.org/IO .1080/10382046.2017.1330039

Arrebola, J.C. \& Martínez, R. (20I7). El cambio climático en los libros de texto españoles de Educación Primaria: Un análisis de las actividades, en Cristina, A. Sande, E. \& Helena, M. (Eds.). VIII Congreso Ibérico de Didáctica da Geografía, (58I-560). Associação de Professores de Geografia.

Arto, M. (2009a). O cambio climático narrado por alumnos de educación secundaria: análise de metáforas e iconas. AmbientalMENTE sustentable, I (7), II5-I25.

Arto, M. (2009b). El cambio climático narrado por alumnos de Educación Primaria y Secundaria: Propuesta de análisis para dibujos y textos, en Mercé Junyent, M. \& Cano, L. (Eds.). Investigar para avanzar en Educación Ambiental. Naturaleza y Parques Nacionales, (II-30). Serie Educación Ambiental.

BeL, J.C. \& Colomer, J.C. (20I8). Teoría y metodología de investigación sobre libros de texto: análisis didáctico de las actividades, las imágenes y los recursos digitales en la enseñanza de las Ciencias Sociales. Revista Brasileira de Educação, 23, I-23. https://doi.org/IO.I590/ SI4I3-247820I8230082

BorG, S. (2003). Teacher cognition in language teaching. A review of research on what teachers think, know, believe, and do. Language Teaching 36, 8I-Io9. https://doi.org/Io.IOI7/ So26I444803001903

BozKURT, F. (20I9). Evaluation of geography textbooks in terms of misconceptions about climate topic. Review of International Geographical Education, 9 (I), I49-I70. https://doi. org/I0.33403/rigeo.573480

CARIDE, J.A. \& MEIRA, P.A. (20I9). Educación, ética y cambio climático. Innovación Educativa, 29, (Ejemplar dedicado a: Respostas educativas innovadoras ao cambio climático; I-II), 6I-76. https://doi.org/IO.I5304/ie.29.6336

CARMAn, J., Zint, M., Burkett, E. \& IBÁÑEz, I. (2020). The role of interest in climate change instruction. Science Education, 105 (2), 309-352. https://doi.org/IO.I002/sce.2I6IO

Centros de Estudios y Experimentación de Obras Públicas (CEDEX) (20I7). Evaluación del impacto del cambio climático en los recursos hídricos y sequías en España. Madrid, Centro de Estudios Hidrográficos, Ministerio de Fomento y Ministerio de Medio Ambiente.

Chang, C.H. \& Pascua, L. (20I6). Singapore students' misconceptions of climate change. International Research in Geographical and Environmental Education, 25 (I), 84-96. https:// doi.org/IO.IO80/10382046.2015.II06206

Clavero, J.M. \& Raso, P. (I983). Los climas: fundamentos y sugerencias didácticas. Editorial Anaya. 
Corrales, M., Moreno, J., SÁnchez, J. \& Zamora, F. (2018). Los paisajes y sus climas. Experiencia classroom de aprendizaje de climogramas y uso de TIC en el aula de $\mathrm{I}^{\mathrm{o}}$ ESO, en García de la Vega, A., (Ed.). Contribución didáctica al aprendizaje de la geografía. Asociación de Geógrafos Españoles y Universidad Autónoma de Madrid.

Cruz, L.A. (2006). Observación meteorológica sin aparatos. Propuesta didáctica de Geografía para el alumnado del programa de diversificación curricular (PDC). Didáctica Geográfica, $8,13-32$.

Cruz, L.A. (2008). Análisis de tipos de nubes como recurso didáctico para el tratamiento interdisciplinar de la Geografía y la Historia del Arte en Ciencias Sociales con alumnos de Educación Secundaria, en Marrón, M.J., Rosado, M.D. \& Rueda, C. (Coord.). Enseñar geografía: la cultura geográfica en la era de la globalización, (20-50).

Cruz, L.A. (20I0). Diseño de un taller de meteorología para alumnos de educación secundaria, en Marrón, M.J. (Coord.). Geografía, educación y formación del profesorado en el marco del espacio europeo de educación superior. Vol. I., (215-236), Grupo de Didáctica de la Geografía de la Asociación de Geógrafos Españoles y Universidad Complutense de Madrid.

Da Rocha, V.T., Brandli, L.L., MazutTi, J., Dal Moro, L., Gasperina, L.D. \& Kalil, R.M.L (2020). Teacher's Approach on Climate Change Education a Case Study, en Leal Filho W., Tortato U. \& Frankenberger F. (Eds). Universities and Sustainable Communities: Meeting the Goals of the Agenda 2030. World Sustainability Series, (617-642). Springer. https://doi. org/I0.1007/978-3-030-30306-8_37

DA SILVA, M.S. \& CARDOSO, C. (20I9). Challenges and perspectives for the teaching geographic Climatology at school. Geosaberes, Io (20), I-I7. https://doi.org/Io.26895/geosaberes. VIoizo.69I

Da Silva, E.M., De Freitas, F.C.C., Neto, L.R.B., Girao, A.F., Pinheiro, C.A., Pinho, L.U. \& Catanho, P.A.G. (20I9). The importance of teaching climatology in civil defense actions in regions of socioeconomic vulnerability of Fortaleza/CE. Revista Brasileira de Meteorologia, 34(3), 369-378. https://doi.org/I0.1590/oI02-7786343045

De Miguel, R. (2013). Geoinformación e innovación en la enseñanza-aprendizaje de la geografía: un reto pendiente en los libros de texto de secundaria. Didáctica de las Ciencias Experimentales y Sociales, 27, 67-90.

De Pro, A. \& EzQuerra, A. (2006). Posibles usos didácticos de los espacios meteorológicos de la televisión. REEC: Revista electrónica de enseñanza de las ciencias, 5 (I), II4-I35.

García de la Vega, A. (20I6). Análisis curricular, rigor científico en los textos literarios sobre las observaciones del tiempo y clima. Propuesta didáctica con fuentes primarias, en Sebastiá, R. \& TondA, E. (Eds.). La investigación e innovación en la enseñanza de la Geografía, (2II-236). Universidad de Alicante.

Gil, A. \& OlcinA, J. (20I7). Tratado de climatología. Servicio de Publicaciones de la Universidad de Alicante.

GonZÁLEZ, E. \& MALDONADO, A. (20I4). ¿Qué piensan, dicen y hacen los jóvenes universitarios sobre el cambio climático?: Un estudio de representaciones sociales. Educar em revista, (3), 35-55. https://doi.org/I0.1590/0104-4060.38106

HANNAH, A. L. \& RhUBART, D. C. (2020). Teacher perceptions of state standards and climate change pedagogy: opportunities and barriers for implementing consensus-informed instruction on climate change. Climate Change, 158, (3-4), 377-392. https://doi.org/I0.1007/ SI0584-0I9-02590-8

Intergovernmental Panel on Climate Change (IPCC) (2014). Climate Change 2013 and Climate Change 2014 (3 vols.). Recuperado en 202I, el 28 de julio, de: http://www.ipcc.ch/ 
Intergovernmental Panel on Climate Change (IPCC) (202I). AR6 Climate Change 2022: Mitigation of Climate Change. Recuperado en 202I, el ro de agosto. https://www.ipcc.ch/ report/sixth-assessment-report-working-group-3/

Jeong, J.S., GonZález-Gómez, D., Conde-Núñez, M.C., SÁnchez-Cepeda, J.S. \& YllanaPrieto, F. (202I). Improving climate change awareness of preservice teachers (Psts) through a university science learning environment. Education Sciences, II(2), I-I7. https:// doi.org/I0.3390/educsciII020078

JodeLET, D. (Ed.). (1989). Les représentations sociales. Presses Universitaires de France.

KhAlidi, R. \& RAMSEY, J. (2020). A comparison of California and Texas secondary science teachers' perceptions of climate change. Environmental Education Research, 27 (5), 669-689. https://doi.org/I0.I080/I3504622.2020.I838447

KURUP, P.M., LEVINSON, R. \& Li, X. (202I). Informed-Decision Regarding Global Warming and Climate Change Among High School Students in the United Kingdom. Canadian Journal of Science, Mathematics and Technology Education, 2I, I66-I85. https://doi.org/I0.1007/ 542330-020-00123-5

Li, C.J., Monroe, M.C., Oxarart, A. \& Ritchie, T. (202I). Building teachers' self-efficacy in teaching about climate change through educative curriculum and professional development. Applied Environmental Education and Communication, 20 (I), 34-48. https:// doi.org/I0.1080/1533015X.2019.1617806

LI, Y.Y. \& Liv, S.C. (202I). Examining Taiwanese students' views on climate change and the teaching of climate change in the context of higher education. Research in Science and Technological Education. https://doi.org/I0.I080/02635143.2020.1830268

MARrón, M.J. (2006). Enseñar y aprender geografía a partir de distintos lenguajes: una experiencia de trabajo por proyectos para estudiar el clima de España a partir de los refranes, en Marrón, M.J., Sánchez, L. \& Jerez, O. (Coords.). Cultura geográfica y educación ciudadana, (459-482).

MARrón, M.J. (20II). Enseñar y aprender Geografía a partir de distintos lenguajes. Una experiencia de trabajo por proyectos para estudiar el clima de España a partir de los refranes. Anales de Geografía, 3 I (I), I07-I23. https://doi.org/I0.5209/rev_AGUC.20II.V3I.nI.5

Martín, L.A. (1984). El clima de nuestra ciudad. Universidad de Zaragoza, Instituto de Ciencias de la Educación.

MartíneZ-Medina, R. (20i6). La enseñanza de la Climatología en el cambio normativo LOE-LOMCE (España): una mirada a través de los Manuales Escolares de Educación Primaria. Iartem E-Journal, 8 (I-2), 50-66.

MarTínez-Romera, D.D. (2013). Situación didáctica y posibilidades del climograma como recurso digital. Didáctica Geográfica, I4, 57-68.

MARTíneZ-FernándeZ, L.C. \& OlCINA, J. (2019). La enseñanza escolar del tiempo atmosférico y del clima en España: currículo educativo y propuestas didácticas. Anales de Geografía, 39 (I), I25-I48. https://doi.org/I0.5209/aguc.64680

Martínez-Medina. R. \& López-Fernández, J.A. (20I6). La enseñanza de la climatología en los manuales escolares de ciencias sociales en Educación Primaria, en Sebastiá, R. \& Tonda, E.M. (Coords.). La investigación e innovación en la enseñanza de la Geografía, (245258). Servicio de Publicaciones de la Universidad de Alicante.

Martínez-lbarra, E., Arias, J. \& Gómez, J. (20i6). La tradición oral como recurso en la enseñanza y aprendizaje del tiempo y el clima. Propuesta didáctica para el clima «Mediterráneo» de la Península Ibérica, en Alanís, L., Almuedo, J., De Oliveira, G., Iglesias, R., Pedregal, B. (Eds.). Nativos digitales y geografía en el siglo XXI: Educación geográfica y 
estilos de aprendizaje, (334-347). Asociación de Geógrafos Españoles. Grupo de Didáctica de la Geografía.

McWhirter, N. \& Shealy, T. (20I8). Case-based flipped classroom approach to teach sustainable infrastructure and decision-making. International Journal of Construction Education and Research, I6 (I), I-2I. https://doi.org/IO.I080/I557877I.20I8.I487892

MolTó, E. (2019). Educación climática: el Museo del Clima de Beniarrés, en Olcina, J. \& Moltó, E.A. (Coords). Climas y tiempos del País Valenciano, (68-7I). Servicio de Publicaciones de la Universidad de Alicante.

Moltó, E. \& Morote, A.F. (20I9). El Museo del Clima de Beniarrés (Alicante). Aprender Geografía y Climatología a través del juego, en Valero, J. \& Fuertes, A. (Eds.). I Congrés d' Humanitats, Ciències Socials i Educació, (5I-59). Publicaciones de la Universidad de Alicante.

Morales-Yago, F.J. (20I6). «Aprendizaje cooperativo y mapas de tiempo: una experiencia de aprendizaje en el aula de I ESO», en Sebastiá, R. \& Tonda, M. E. (Eds.). Investigar para innovar en la enseñanza de la Geografía, (252-266). Servicio de Publicaciones de la Universidad de Alicante.

Moreno-Vera, J.R. (2020). Wall-e en el aula de Ciencias Sociales. Trabajar el cambio climático a través del cine post-apocalíptico. Making Off. Cuadernos de cine y educación, (I5I), I9-24.

Morote, A.F. (2020a). ¿Cómo se trata el tiempo atmosférico y el clima en la Educación Primaria? Una exploración a partir de los recursos y actividades de los manuales escolares de Ciencias Sociales. Espacio, Tiempo y Forma, Serie VI Geografía, I3, II9-I44.

Morote, A.F. (2020b). La investigación sobre manuales escolares de Geografía españoles: Análisis bibliométrico (1980-2019). Anales de Geografía, 40 (2), 467-497. http://dx.doi. org/I0.5209/AGUC.72983

Morote, A.F. \& Hernández, M. (2020). Social Representations of Flooding of Future Teachers of Primary Education (Social Sciences): A Geographical Approach in the Spanish Mediterranean Region. Sustainability, I2 (I5), I-I4. https://doi.org/I0.3390/suI2I56065

Morote, A.F. \& Moltó, E. (20I7). El Museo del Clima de Beniarrés (Alicante). Propuesta de un recurso didáctico para la enseñanza de la Climatología. Didáctica de las Ciencias Experimentales y Sociales, 32 (I), I09-I3I. https://doi.org/I0.7203/dces.32.9624

Morote, A.F. \& Olcina, J. (2020). El estudio del cambio climático en la Educación Primaria: una exploración a partir de los manuales escolares de Ciencias Sociales de la Comunidad Valenciana. Cuadernos Geográficos, 59 (3), 5-24. http://dx.doi.org/I0.30827/cuadgeo. v59i3.II792

Morote, A. F. \& Olcina, J. (202Ia). Riesgos atmosféricos y cambio climático: propuestas didácticas para la región mediterránea en la enseñanza secundaria. Investigaciones Geográficas, (76), 195-220. https://doi.org/I0.I4I98/INGEO.I8510

Morote, A. F. \& Olcina, J. (202rb). Cambio climático y sostenibilidad en la Educación Primaria. Problemática y soluciones que proponen los manuales escolares de Ciencias Sociales. Sostenibilidad: económica, social y ambiental, 3, 25-43. https://doi.org/I0.I4I98/ Sostenibilidad2021.3.02

Morote, A.F. \& Souto X. M. (2020). Educar para convivir con el riesgo de inundación. Estudios Geográficos, 8I (288), I-I4. https://doi.org/I0.3989/estgeogr.20205I.03I

Morote, A.F., CAMPO, B.A. \& Colomer, J.C. (20I9). La percepción del cambio climático en los futuros docentes de Educación Primaria. Una experiencia de conocimientos previos a partir de la enseñanza de las Ciencias Sociales, en AGE y Universidad de Valencia. Crisis y espacios de oportunidad. Retos para la Geografía, (I06-I20). Universidad de Valencia.

Moscovicı, S. (1978). La psychanalyse, son image, son public. Presses Universitaires de France. 
MuIs, K.R. \& DufFY, M.C. (2013). Epistemic climate and epistemic change: Instruction designed to change students' beliefs and learning strategies and improve achievement. Journal of Educational Psychology, I05(I), 213-225. https://doi.org/I0.1037/a0029690

NAMDAR, B. (2018). Teaching global climate change to pre-service middle school teachers through inquiry activities. Research in Science and Technological Education, 36(4), 440-462. https://doi.org/IO.I080/02635143.2017.I420643

Navalón, A.M. (1997). El viaje climatológico. Aula de Innovación Educativa, 62, 74-77.

Navarro-Díaz, M., Moreno, O. \& Rivero, A. (2020). El cambio climático en los libros de texto de educación secundaria obligatoria. Revista Mexicana de Investigación Educativa, 25 (87), 957-985.

Olcina, J. (2014). Enseñanzas climáticas en la obra de Kant. Anales de Geografía de la Universidad Complutense, 34 (2), II9-I62. https://doi.org/I0.5209/rev_AGUC.20I4.v34. $n 2.47075$

OlciNA, J. (2017). La enseñanza del tiempo atmosférico y del clima en los niveles educativos no universitarios. Propuestas didácticas, en Sebastiá, R. \& Tonda, E.M. (Eds.). Enseñanza y aprendizaje de la Geografía para el siglo XXI (II9-I48). Servicio de Publicaciones de la Universidad de Alicante.

OlCINA, J. \& ZAMORA, R. (2000). Los riesgos naturales a través de la red Internet. Recursos didácticos y de investigación. Boletín de la Asociación de Geógrafos Españoles, 30, 193-205. https://bage.age-geografia.es/ojs/index.php/bage/article/view/372

PAGÈs, J. (2008). Los libros de texto de ciencias sociales, geografía e historia y el desarrollo de las competencias ciudadanas, en Seminario Internacional Textos escolares de historia y ciencias sociales, (24-56). Ministerio de Educación de Chile.

Pérez-Morales, A., Gil, S. \& Quesada (202I). Do we all stand equally towards the flood? Analysis of social vulnerability in the Spanish Mediterranean coast. Boletín de la Asociación de Geógrafos Españoles, (88), I-39. https://doi.org/I0.2II38/bage.2970

Ramiro, E. (2006). Una Isla que se mueve. Aproximación a la isla de calor urbana de Algemesí (Valencia) y su aprovechamiento didáctico. Didáctica Geográfica, 8, 33-50.

Ramos, J.M. \& CAlONGE, G. (20I4). Renovación de la didáctica de la Geografía física en segundo de bachillerato: La climatología como ejemplo. Didáctica Geográfica, I5, I29-I5I.

RepetTo, E. (1984). Una experiencia de didáctica de la física: estudio del tiempo meteorológico. El Guiniguada, I, 23-3I.

Rodrigo, A. (2006). El lenguaje cartográfico y la enseñanza del clima: Un análisis de los mapas en los libros de texto de España y Brasil. Didáctica Geográfica, 8, 5I-68.

Rubio, S. \& Martínez, R. (20I4). ¿Es competente el alumnado del Grado de Educación Primaria en la comprensión del tiempo atmosférico y el clima?, en Martínez, R. \& Tonda, E.M. (Coords.). Nuevas perspectivas conceptuales y metodológicas para la educación geográfica, (I33-I52). Grupo de Didáctica de la Geografía de la AGE y Universidad de Córdoba.

SÁnCHEZ-Ogallar, A. (2000). El cambio climático como ejemplo de trasposición didáctica compleja. En González, J.L. \& Marrón, M.J. (Eds.). Geografía, Profesorado y Sociedad. Teoría y práctica de la Geografía en la enseñanza, (483-496). Asociación de Geógrafos Españoles.

SÁNCHEZ-OgALlAR, A. (2005a). Una propuesta didáctica para la enseñanza del clima en la Educación Secundaria Obligatoria, en Sande, E. (Coord.). Ensinar Gegrafia na sociedade do conhecimento (23I-244). Asociación de Geógrafos Españoles y Associaçao de Profesores de Geografia de Portugal.

SÁNCHEZ-OgALlaR, A. (2005b). Una propuesta didáctica para la enseñanza del clima en la Educación Secundaria Obligatoria, en Ensinar Geografía na sociedade do conhecimento, (23I-244). Asociación Española de Geografía. 
Sebastiá, R. \& Tonda, E. M. (20i2). Características y evolución de la Revista Didáctica Geográfica. Didáctica Geográfica, I2, I9-48.

Sebastiá, R. \& TONDA E.M. (20I8). Enseñar y aprender el tiempo atmosférico y clima, en García de la Vega, A. (Coord.). Reflexiones sobre educación geográfica: revisión disciplinar e innovación didáctica, (153-I76). Asociación Española de Geografía.

Serantes, A. (2015). Como abordan o Cambio Climático os libros de texto da Ensinanza Secundaria Obligatoria na España. AmbientalMENTEsustentable, 20 (2015), 249-262. doi I0.17979/ams.2015.2.20.1609.1603

SeZEn-BARRie, A. \& MARBACH-Ad, G. (202I). Cultural-historical analysis of feedback from experts to novice science teachers on climate change lessons. International Journal of Science Education, 43 (4), 497-528. https://doi.org/I0.1080/09500693.2020.1870759

SoÑora, F., Rodríguez, M.M. \& Troitiño, R. (2009). Un modelo activo de educación ambiental: Prácticas sobre cambio climático. Enseñanza de las Ciencias de la Tierra, I7 (2), 196-206.

Souto, X.M. (2000). La geografía en los libros de texto de la Enseñanza Secundaria. Debate sobre la edición del informe de la AGE, elaborado por Jacobo García y Daniel Marías, así como la carta de la profesora $\mathrm{M}^{\mathrm{a}}$ Luisa de Lázaro (2000). Asociación Española de Geografía.

Souto, X. M. (2018). La geografía escolar: deseos institucionales y vivencias de aula. Boletín de la Asociación de Geógrafos Españoles, 79, I-3I. http://dx.doi.org/I0.2II38/bage.2757

Tonda, E. \& Sebastiá. R. (2003). Las dificultades en el aprendizaje de los conceptos de tiempo atmosférico y clima: la elaboración e interpretación de climogramas. Revista de Educación de la Universidad de Granada, 16, 47-69.

TuCKer, L. \& Sherwood, L. (2020). Entender el Cambio Climático. Información y Recursos para el Profesorado. Narcea.

United Nations (2015). Sustainable Development Goals. UNDP, Sustainable Development Agenda. Recuperado en 202I, el 28 de julio, de: https://www.undp.org/content/undp/es/ home/sustainable-development-goals/resources.html

Valbuena, M. \& VAlverde, J.A. (2006). La Climatología Local: Procedimiento para su enseñanza y aprendizaje. Didáctica Geográfica, 8 (2006), 93-ı08.

WiSE, S.B. (20I0). Climate change in the classroom: Patterns, motivations, and barriers to instruction among Colorado science teachers. Journal of Geoscience Education, 58(5), 297-309. https://doi.org/10.5408/1.3559695

Yow, D.M. (2014). Teaching introductory weather and climate using popular movies. Journal of Geoscience Education, 62(I), II8-I25. https://doi.org/I0.5408/I3-OI4.I 
ANEXO I. LISTA COMPLETA DE TRABAJOS IDENTIFICADOS SOBRE LA INVESTIGACIÓN
Y ENSEÑANZA EN DIDÁCTICA DE LA CLIMATOLOGÍA EN ESPAÑA (1980-2020)

\begin{tabular}{|c|c|c|}
\hline $\begin{array}{l}\text { AUTORES/AS Y AÑO } \\
\text { DE PUBLICACIÓN }\end{array}$ & EJE & TEMÁTICA \\
\hline Campo \& García (2020) & Clima & Representaciones sociales \\
\hline García-Vinuesa et al. (2020) & Cambio climático & Representaciones sociales \\
\hline González et al. (2020) & Cambio climático & Enseñanza \\
\hline Moreno-Vera (2020) & Cambio climático & Enseñanza \\
\hline Morote (2020a) & Clima & Libros de texto \\
\hline Morote (2020b) & Cambio climático & Libros de texto \\
\hline Morote (2020c) & Cambio climático & Representaciones sociales \\
\hline Morote \& Hernández (2020a) & Cambio climático & Enseñanza \\
\hline Morote \& Hernández (2020b) & Cambio climático & Representaciones sociales \\
\hline Morote \& Olcina (2020) & Cambio climático & Libros de texto \\
\hline Morote \& Souto (2020) & Cambio climático & Representaciones sociales \\
\hline Navarro et al. (2020) & Cambio climático & Libros de texto \\
\hline Robredo \& Ladreda (2020) & Cambio climático & Representaciones sociales \\
\hline Varela et al. (2020) & Cambio climático & Representaciones sociales \\
\hline Velasco et al. (2020) & Cambio climático & Representaciones sociales \\
\hline Caride \& Meira (2019) & Cambio climático & Enseñanza \\
\hline Ferrari et al. (2019) & Cambio climático & Representaciones sociales \\
\hline García-Vinuesa et al. (2019) & Cambio climático & Representaciones sociales \\
\hline García et al. (2019) & Cambio climático & Representaciones sociales \\
\hline Gómez \& Corrochano (2019) & Cambio climático & Enseñanza \\
\hline López \& Guerrero (2019) & Cambio climático & Representaciones sociales \\
\hline $\begin{array}{l}\text { Martínez-Fernández \& Olcina } \\
\text { (2019) }\end{array}$ & Clima & Libros de texto \\
\hline Moltó (2019) & Clima & Enseñanza \\
\hline Moltó \& Morote (2019) & Clima & Enseñanza \\
\hline Morote (2019a) & Cambio climático & Enseñanza \\
\hline Morote (2019b) & Cambio climático & Representaciones sociales \\
\hline Morote (2019c) & Cambio climático & $\begin{array}{l}\text { Libros de texto/ } \\
\text { representaciones sociales }\end{array}$ \\
\hline Morote et al. (2019) & Cambio climático & Representaciones sociales \\
\hline Ormaetxea et al. (2019) & Cambio climático & Enseñanza \\
\hline Roldán et al. (2019) & Cambio climático & Representaciones sociales \\
\hline Sánchez-Emeterio (2019) & Cambio climático & Enseñanza \\
\hline Abarca et al. (2018) & Clima & Enseñanza \\
\hline Corrales et al. (2018) & Clima & Enseñanza \\
\hline Gónzález y Arias (2018) & Clima & Enseñanza \\
\hline
\end{tabular}




\begin{tabular}{|c|c|c|}
\hline Meira et al. (2018) & Cambio climático & Representaciones sociales \\
\hline Roldán et al. (2018) & Cambio climático & Representaciones sociales \\
\hline Sanchis et al. (2018) & Cambio climático & Representaciones sociales \\
\hline Sebastiá \& Tonda (2018) & Clima & Enseñanza \\
\hline Arrebola \& Martínez (2017) & Cambio climático & Libros de texto \\
\hline Benavides et al. (2017) & Cambio climático & Representaciones sociales \\
\hline Campo \& Martínez (2017) & Clima & Enseñanza \\
\hline $\begin{array}{l}\text { Martínez-Fernández \& Olcina } \\
\text { (2019) }\end{array}$ & Clima & Enseñanza \\
\hline Morote \& Moltó (2017) & Clima & Enseñanza \\
\hline Olcina (2017) & Clima/cambio climático & Libros de texto \\
\hline Ouriachi et al. (2017b) & Cambio climático & Enseñanza \\
\hline Ouriachi et al. (2017b) & Cambio climático & Enseñanza \\
\hline García de la Vega (2016) & Clima & Enseñanza \\
\hline Martínez-Medina (2016) & Clima & Libros de texto \\
\hline $\begin{array}{l}\text { Martínez-Medina \& López- } \\
\text { Fernández (2016) }\end{array}$ & Clima & Libros de texto \\
\hline Martínez et al. (2016) & Clima & Enseñanza \\
\hline Morales-Yago (2016) & Clima & Enseñanza \\
\hline Morote (2016) & Clima & Enseñanza \\
\hline $\begin{array}{l}\text { Méndez y Martínez-Martínez } \\
\text { (2015) }\end{array}$ & Clima & Enseñanza \\
\hline Serantes (2015) & Cambio climático & Libros de texto \\
\hline Domenech-Casal (2014) & Cambio climático & Enseñanza \\
\hline Grasso (2014) & Clima & Enseñanza \\
\hline Olcina (2014) & Clima & Enseñanza \\
\hline Ramos \& Calonge (2014) & Clima & Libros de texto \\
\hline $\begin{array}{l}\text { Rubio \& Martínez-Medina } \\
\text { (2014) }\end{array}$ & Clima & $\begin{array}{l}\text { Enseñanza/ } \\
\text { representaciones sociales }\end{array}$ \\
\hline Martínez-Romera (2013) & Clima & Enseñanza \\
\hline Genovart (2012) & Clima & Enseñanza \\
\hline $\begin{array}{l}\text { García-Rodeja \& De Oliveira } \\
(2012)\end{array}$ & Cambio climático & Representaciones sociales \\
\hline Marrón (2011) & Clima & Enseñanza \\
\hline Punter et al. (2011) & Cambio climático & Representaciones sociales \\
\hline Cruz (2010) & Clima & Enseñanza \\
\hline Romero (2010) & Clima & Enseñanza \\
\hline Gómez et al. (2010) & Cambio climático & Enseñanza \\
\hline Arto (2009a) & Cambio climático & Representaciones sociales \\
\hline Arto (200gb) & Cambio climático & Representaciones sociales \\
\hline
\end{tabular}




\begin{tabular}{|l|l|l|}
\hline González y Meira (2009) & Cambio climático & Enseñanza \\
\hline Soñora et al. (2009) & Cambio climático & Enseñanza \\
\hline Cruz (2008) & Clima & Enseñanza \\
\hline Cruz (2006) & Clima & Enseñanza \\
\hline De Pro \& Ezquerra (2006) & Clima & Enseñanza \\
\hline López-Martín (2006) & Clima & Enseñanza \\
\hline Marrón (2006) & Clima & Enseñanza \\
\hline Ramiro (2006) & Clima & Enseñanza \\
\hline Rodrigo (2006) & Clima & Enseñanza/libros de texto \\
\hline Vabuena \& Valverde (2006) & Clima & Representaciones sociales \\
\hline Sánchez-Ogallar (2005a) & Clima & Enseñanza \\
\hline Sánchez-Ogallar (2005b) & Clima & Enseñanza \\
\hline Tonda \& Sebastiá (2003) & Clima & $\begin{array}{l}\text { Enseñanza/ } \\
\text { representaciones sociales }\end{array}$ \\
\hline Juan \& Molina (2001) & Clima & Enseñanza \\
\hline Olcina \& Zamara (2000) & Cambio climático & Enseñanza \\
\hline Sánchez-Ogallar (2000) & Cambio climático & Enseñanza \\
\hline Navalón (1997) & Clima & Enseñanza \\
\hline Martín (1984) & Clima & Enseñanza \\
\hline Repetto (1984) & Clima & Enseñanza \\
\hline Clavero \& Raso (1983) & Clima & Enseñanza \\
\hline
\end{tabular}

Elaboración propia 
ANEXO II. PÁGINAS WEBS DONDE SE HAN CONSULTADO Y ESTÁN DISPONIBLES DIFERENTES TRABAJOS SOBRE DIDÁCTICA DE LA CLIMATOLOGÍA

-Revista Didáctica Geográfica (trabajos disponibles desde 1977): https://didacticageografica.age-geografia.es//index.php/didacticageografica

- Congresos organizados por el Grupo de Didáctica de la Geografía (AGE) (trabajos disponibles desde 1988): http://didacticageografia.age-geografia.es//index.php? $m=4$

-Congresos de la Asociación Española de Geografía (AGE) (trabajos disponibles desde 2013): https://www.age-geografia.es/site/publicaciones-no-periodicas/\#

- Congresos Ibéricos de Geografía (AGE) y Asociación Portuguesa de Geografía (APG) (trabajos disponibles en la web desde 2002):

https://www.age-geografia.es/site/publicaciones-no-periodicas/\#

-Congresos de la Unión Geográfica Internacional (UGI) (trabajos disponibles en la web desde 1998): https://www.age-geografia.es/site/publicaciones-no-periodicas/\#

-Congresos de la Asociación Universitaria de Profesorado de Didáctica en las Ciencias Sociales (AUPDCS) (trabajos disponibles en la web desde 1997):

http://didactica-ciencias-sociales.org/publicaciones/libros/

Elaboración propia 


\section{4

\section{Presentación · Foreword}

\section{Artículos · Articles}

19 Eugenio Climent López y Samuel Esteban Rodríguez

La crisis económica de 2008 en las Denominaciones de Origen Protegidas de vino españolas: efectos y modelos de salida. The 2008 Economic Crisis in the Spanish Protected Designations of Origin of Wine: Effects and Exit Models

4.5 David García-Reyes, EnRICo Marini y Marta Gallardo

Infancia, pobreza y deporte en el desierto de Calama, Chile. Representaciones sociales en «Segundo tiempo, último gol gana» . Childhood, Poverty and Sport in the Desert of Calama, Chile. Social Representations in «Segundo tiempo, último gol gana»

José Antonio González díaz, Benjamín González díaz y Rocío Rosa GARCía

Revisión de los objetivos de los espacios protegidos: el parque natural y reserva de la biosfera de Redes - Review of the Objectives of Protected Spaces: Natural Park and Biosphere Reserve of Redes

Álvaro-Francisco Morote Seguido

Investigación y enseñanza en didáctica de la climatología. Estado de la cuestión (1980-2020) (España) · Research and Teaching in Didactic of Climatology. State of the Art (1980-2020) (Spain)

\section{Gemma Ruiz Ángel y Mariano Cecilia Espinosa}

Rogativas pro - pluvia en la catedral de Orihuela: La sequía en Orihuela durante el siglo XVII · Rogative Pro - Pluvia in the Cathedral of Orihuela: The Drought in Orihuela during the $17^{\text {th }}$ Century

135 José Ramón Sánchez Holgado

La puesta en valor de los campos de concentración y exterminio nazis como lugar de memoria . Putting Value of the Nazis Extermination and Concentration Camps as a Place of Memory

\section{7}

Laura Sevilla Cuadrado

La conciencia medio ambiental en el sector del turismo de sol y playa. Un estudio con la técnica Delphi . Environmental Awareness in the Sun, Sea and Sand Tourism Sector. A Study using the Delphi Technique

The Technopolitics of Mapping Dar es Salaam: An Examination of the Technological and Political Motivations of the Humanitarian OpenStreetMap Team La tecnopolítica de la cartografía de Dar es Salaam: Un examen de las motivaciones tecnológicas y políticas del equipo humanitario OpenStreetMap

\section{Reseñas · Book Review}

19 De LÁzaro Torres, María Luisa y Morales Yago, Francisco José (2021). Introducción a la Investigación en Geografía. Pautas para la Elaboración de Trabajos Científicos (An Introduction to Research in Geography. Guidelines for the Preparation of Scientific Papers). UNED, 223 Pp., ISBN: 978-84-362-7689-3. (JuLIO FERNÁNDEZ PORTELA

Fernández Portela, Julio y Hernández García, Ricardo (2021). Sociedad y territorio en Cubillas de Santa Marta: del Catastro de Ensenada a la actualidad. Ayuntamiento de Cubillas de Santa Marta, 259 pp., ISBN: 978-84-09-28428-3. (MIGUEL ÁngEL GarCía VELASCO)

MÉndez GutiérRez del VALLe, R. (2020). Sitiados por la pandemia. Del colapso a la reconstrucción: apuntes geográficos. Revives, 183 pp. (ROSA MECHA

23 MARTín-Roda, Eva María (2021). Geopolítica de los recursos energéticos. Síntesis, 276 pp., ISBN: 9788413571348 ; ISBN Digital: 9788413576626 (AURELIO NiETO CODINA)

235 Moreno BorrelL, S. (2019). La Naturaleza y el Paisaje de Málaga a través de viajeros, naturalistas y científicos. La Serranía, 224 Pp., ISBN: 978-8415588-28-3 (SERGIO REYES CORREDERA)

\section{Imágenes y palabras · Pictures and Words}

\subsection{Julıo López-DavaliLlo LARREA}

La laguna de Herramélluri. Un humedal renacido . The Herramélluri Lagoon. A Wetland Reborn

\section{Síntesis de Tesis Doctorales - Summaries of Doctoral Thesis}

\section{Javier Álvarez Otero}

El uso educativo de las Infraestructuras de Datos Espaciales (IDE) para mejorar la responsabilidad social de los ciudadanos del siglo XXI sobre el territorio. Departamento de Didáctica de las Ciencias Experimentales, Sociales y Matemáticas. Universidad Complutense de Madrid. Directora: Dra. Doña María Luisa de Lázaro Torres. Fecha: 10 de diciembre de 2020

\section{José Fernández Álvarez}

Dinámica evolutiva de los usos y coberturas del suelo en la provincia de Salamanca. Análisis comparado y repercusiones en el paisaje rural de cuatro comarcas de estudio. Departamento de Geografía, Universidad Nacional de Educación a Distancia (UNED). Director: Dr. D. Carlos Javier Pardo Abad. Fecha: 27 de mayo de 2021 